\title{
Metal production in M 33: space and time variations ^
}

\author{
L. Magrini ${ }^{1}$, L. Stanghellini ${ }^{2}$, E. Corbelli ${ }^{1}$, D. Galli ${ }^{1}$, and E. Villaver ${ }^{3}$ \\ 1 INAF - Osservatorio Astrofisico di Arcetri, Largo E. Fermi 5, 50125 Firenze, Italy \\ e-mail: [laura; edvige;galli]@arcetri.astro.it \\ 2 National Optical Astronomy Observatories, Tucson, AZ 85719, USA \\ e-mail: lstanghellini@noao.edu \\ 3 Universidad Autónoma de Madrid, Departamento de Física Teórica C-XI, 28049 Madrid, Spain \\ e-mail: eva.villaver@uam.es
}

Received 28 October 2009 / Accepted 18 December 2009

\section{ABSTRACT}

\begin{abstract}
Context. Nearby galaxies are ideal places to study metallicity gradients in detail and their time evolution.
Aims. We analyse the spatial distribution of metals in M 33 using a new sample and the literature data on $\mathrm{H}$ II regions, and constrain a model of galactic chemical evolution with $\mathrm{H}$ II region and planetary nebula (PN) abundances.

Methods. We consider chemical abundances of a new sample of $\mathrm{H}$ II regions complemented with previous data sets. We compared $\mathrm{H}$ II region and PN abundances obtained with a common set of observations taken at MMT. With an updated theoretical model, we followed the time evolution of the baryonic components and chemical abundances in the disk of M 33, assuming that the galaxy is accreting gas from an external reservoir.

Results. From the sample of $\mathrm{H}$ II regions, we find that i) the 2D metallicity distribution has an off-centre peak located in the southern arm; ii) the oxygen abundance gradients in the northern and southern sectors, as well as in the nearest and farthest sides, are identical within the uncertainties, with slopes around $-0.03-4 \mathrm{dex} \mathrm{kpc}^{-1}$; iii) bright giant $\mathrm{H}$ II regions have a steeper abundance gradient than the other $\mathrm{H}$ II regions; iv) $\mathrm{H}$ II regions and PNe have $\mathrm{O} / \mathrm{H}$ gradients very close within the errors; v) our updated evolutionary model is able to reproduce the new observational constraints, as well as the metallicity gradient and its evolution.

Conclusions. Supported by a uniform sample of nebular spectroscopic observations, we conclude that i) the metallicity distribution in M 33 is very complex, showing a central depression in metallicity probably due to observational bias; ii) the metallicity gradient in the disk of M 33 has a slope of $-0.037 \pm 0.009 \mathrm{dex} \mathrm{kpc}^{-1}$ in the whole radial range up to $\sim 8 \mathrm{kpc}$, and $-0.044 \pm 0.009 \mathrm{dex}^{\mathrm{kpc}}{ }^{-1}$ excluding the central kpc; iii) there is little evolution in the slope with time from the epoch of PN progenitor formation to the present.
\end{abstract}

Key words. galaxies: abundances - galaxies: evolution - galaxies: individual: M 33

\section{Introduction}

Understanding the chemical evolution of nearby galaxies has become more and more interesting in the recent past, given the wealth of new, detailed data available for their stellar and gaseous components. M 33 (NGC 598) is an ideal laboratory for testing chemical evolution models. As a nearby blue starforming galaxy, with a large angular size (optical size $53^{\prime} \times 83^{\prime}$, Holmberg 1958), and an intermediate inclination $\left(i=53^{\circ}\right)$, it is one of the galaxies observed with the greatest resolution and sensitivity. Distance estimates range from $730 \mathrm{kpc}$ (Brunthaler et al. 2005) to $964 \mathrm{kpc}$ (Bonanos et al. 2006). In this paper we adopt an average value of $840 \mathrm{kpc}$ (Freedman et al. 1991). Many spectroscopic studies are available, aiming at determining the physical and chemical properties of its $\mathrm{H}$ II regions (cf., e.g., Smith 1975; Kwitter \& Aller 1981; Vílchez et al. 1988; Willner \& Nelson-Patel 2002; Crockett et al. 2006; Magrini et al. 2007a; Rosolowsky \& Simon 2008; Rubin et al. 2008). These $\mathrm{H}$ II regions trace the present interstellar medium (ISM) and

* Full Tables 2 and 3 are only available in electronic form at the CDS via anonymous ftp to cdsarc.u-strasbg. fr (130.79.128.5) or via

http: //cdsweb.u-strasbg.fr/cgi-bin/qcat?J/A+A/512/A63 their metallicity is a measure of the star formation rate (SFR) integrated over the whole galaxy lifetime. As a result, the metallicity of $\mathrm{H}$ II regions gives interesting constraints to galactic chemical evolution models.

In the past, studies of the metallicity gradient of $\mathrm{H}$ II regions in M 33 have shown a very steep profile. First quantitative spectroscopic studies were carried out by Smith (1975), Kwitter \& Aller (1981) and Vílchez et al. (1988). Their observations, limited to the brightest and largest $\mathrm{H}$ II regions, led to a radial oxygen gradient about $-0.1 \mathrm{dex} \mathrm{kpc}^{-1}$. Considering the observations by the above researchers, Garnett et al. (1997) obtained an overall $\mathrm{O} / \mathrm{H}$ gradient of $-0.11 \pm 0.02 \mathrm{dex} \mathrm{kpc}-1$.

With increasing sample sizes, flatter gradients have been determined, in particular by i) Willner \& Nelson-Patel (2002) who derive neon abundances of $25 \mathrm{H}$ II regions from infrared lines, obtaining a Ne/H gradient of $-0.034 \pm 0.015 \mathrm{dex} \mathrm{kpc}{ }^{-1}$; ii) Crockett et al. (2006) who derive even shallower gradients $\left(-0.016 \pm 0.017 \mathrm{dex} \mathrm{kpc}^{-1}\right.$ for $\mathrm{Ne} / \mathrm{H}$ and $-0.012 \pm$ $0.011 \mathrm{dex} \mathrm{kpc}^{-1}$ for $\mathrm{O} / \mathrm{H}$ ) from optical spectra of $6 \mathrm{H}$ II regions; iii) Magrini et al. (2007a) who obtain an $\mathrm{O} / \mathrm{H}$ gradient of $14 \mathrm{H}$ II regions, located in the radial range from $\sim 2$ to $\sim 7.2 \mathrm{kpc}$ with a slope of $-0.054 \pm 0.011 \mathrm{dex} \mathrm{kpc}^{-1}$; iv) Rosolowsky \& Simon (2008) who observe the largest sample of H II regions, 61, 


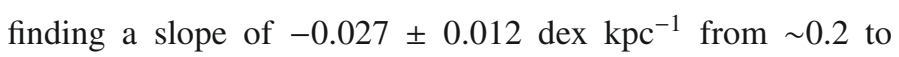
$\sim 6 \mathrm{kpc}$; v) Rubin et al. (2008) who obtain Ne/H and S/H gradients from Spitzer infrared spectra of $-0.058 \pm 0.014 \mathrm{dex} \mathrm{kpc}{ }^{-1}$ and $-0.052 \pm 0.021 \mathrm{dex} \mathrm{kpc}^{-1}$, respectively.

Rosolowsky \& Simon (2008) attribute the large discrepancies in different authors' determinations to an intrinsic scatter of about 0.1 dex around the average gradient, but the scatter is unexplained by the abundance uncertainties. This kind of scatter is also seen in the Milky Way gradient from H II regions (e.g. Afflerbach et al. 1997), and it may be caused by metallicity fluctuations in the ISM and by the spiral arms. Thus a limited number of observations, coupled with a significant metallicity scatter at a given radius, may produce widely varying results. In the case of a shallow gradient this effect is even stronger; for example, for an abundance gradient of -0.02-0.03 dex kpc-1 in a galaxy with a radius of $10 \mathrm{kpc}$ and a scatter of $0.1 \mathrm{dex}$, one would need more than $30 \mathrm{H}$ II regions to obtain a good estimate of the slope of the gradient (Bresolin et al. 2009). Thus, only a large number of measurements can overcome the uncertainties engendered by the intrinsic variance and relatively shallow gradient of M 33.

Our sample is composed of $48 \mathrm{H}$ II regions. We derived the physical and chemical properties for $19 \mathrm{H}$ II regions which have not been observed previously, and for other $14 \mathrm{H}$ II regions whose chemical abundances have already been published. For the remaining $\mathrm{H}$ II regions, the faintness of their spectra did not allow any reliable abundance determination, since their electron temperature $\left(T_{\mathrm{e}}\right)$ could not be derived. We complemented these observations by a sample of 102 planetary nebulae (PNe), already presented by Magrini et al. (2009a, hereafter M09), observed during the same run. The main advantage of observing a combined sample of $\mathrm{H}$ II regions and $\mathrm{PNe}$ is being able to use not only the same observational set-up, but also the same data reduction and analysis techniques, and to use identical abundance determination methods.

Although our sample of $\mathrm{H}$ II regions does not add much to the literature, the presence of several objects in common with previous studies allows us to check the consistency of different sets of chemical abundance results. By including at the same time two stellar populations of different ages but with similar spectroscopic characteristics, our observations allowed us to study for the first time the global metallicity, its 2-dimensional (2D) distribution and its radial gradient, at two different epochs in the galaxy's lifetime avoiding biases introduced by different metallicity analysis. The aim of the present study is to settle the questions of the value of the metallicity gradient in M 33 and its time evolution. In this framework, the new observations of $\mathrm{H}$ II regions and $\mathrm{PNe}$ in $\mathrm{M} 33$ complemented with the previous data represent the largest metallicity database available for an external galaxy.

In addition to metallicity data, recent results, such as the detection of inside-out growth in the disk of M 33 (Williams et al. 2009), the detailed analysis of the star formation both in the whole disk (Verley et al. 2009) and in several giant H II regions (Relano \& Kennicutt 2009), stimulated us to revise the already existing chemical evolution model (M07b) and the star formation process in M 33. Particular attention was put on the observational constraints that our previous model failed to reproduce, such as the radial profile of the molecular gas and the relationship between the SFR and the molecular gas.

The paper is organized as follows. In Sect. 2 we describe our sample of H II regions observed with MMT. These data, together with a large literature dataset, allowed us to compute the metallicity gradient of $\mathrm{H}$ II regions again. In Sect. 3 we present the $2 \mathrm{D}$ distribution of the metallicity and the radial gradient of different types of $\mathrm{H}$ II regions. In Sect. 4 we discuss the offcentre metallicity peak and its origin. In Sect. 5 the data are compared with the prediction of chemical evolution model of M 33. Finally, our conclusions and a summary are given in Sect. 6.

\section{The $\mathrm{H}$ ॥ region dataset}

Hot O-B stars ionize their surrounding medium, producing the characteristic emission-line spectra of $\mathrm{H}$ II regions. The $\mathrm{H}$ II regions of $\mathrm{M} 33$ studied in the literature span a wide range of luminosities. Their intrinsic brightness led giant $\mathrm{H}$ II regions to be preferred in the earlier studies (e.g. Kwitter \& Aller 1981; Vilchez et al. 1988) when only relatively small telescopes were available. Smaller and fainter H II regions have instead been the subject of later spectroscopic investigations (e.g., Magrini et al. 2007a; Rosolowsky \& Simon 2008). The latest abundance determinations have been restricted to the H II regions with available electron temperature measurements. Several emission-line diagnostics of nebular $T_{\mathrm{e}}$ are indeed present in the optical spectrum of an H II region, namely: [O III] $4363 \AA$, [N II] $5755 \AA$, [S III] $6312 \AA$, [O II] 7320-7330 А. Determining $T_{\mathrm{e}}$ is the only way to derive the ionic and total chemical abundances safely and accurately. An assumed $T_{\mathrm{e}}$ could produce error of a factor of 2 or more in the final chemical abundances (cf., e.g., Osterbrock \& Ferland 1989). This is why in the following analysis we include only those $\mathrm{H}$ II regions whose $T_{\mathrm{e}}$ is directly measured.

\subsection{The MMT observations: data reduction and analysis}

In November 2007, we obtained spectra of $48 \mathrm{H}$ II regions (and 102 PNe) in M 33 using the MMT Hectospec fiber-fed spectrograph (Fabricant et al. 2005). The spectrograph was equipped with an atmospheric dispersion corrector and it was used with a single setup: $270 \mathrm{~mm}^{-1}$ grating at a dispersion of $1.2 \AA$ pixel $^{-1}$. The resulting total spectral coverage ranged from approximately $3600 \AA$ to $9100 \AA$, thus including the basic emission-lines necessary for determining their physical and chemical properties. The instrument deploys 300 fibers over a 1-degree diameter field of view, and the fiber diameter is $\sim 1.5^{\prime \prime}$ ( 6 pc adopting a distance of $840 \mathrm{kpc}$ to $\mathrm{M} 33$ ).

Some of the $\mathrm{H}$ II regions in our sample already have published spectra in the literature so we use them as control sample, while several are new. In Table 1 we list the $H$ II regions from the total observed sample for which we can derive the physical and chemical properties. The identification names are from: BCLMP - Boulesteix et al. (1974); CPSDP - Courtes et al. (1987); GDK99 - Gordon et al. (1996); EPR2003 - Engargiola et al. (2003); MJ98 - Massey \& Johnson (1998). The H II regions not identified in previous works are labled with LGC-HII-n as in Magrini et al. (2007a), standing for H II regions discovered by the Local Group Census project (cf. Corradi \& Magrini 2006). The coordinates $\mathbf{J} 2000.0$ of the position of the fibers projected on the sky are shown in the third and forth columns. They do not correspond exactly to the centre of the emission line objects, but generally to the maximum [O III] emissivity.

The spectra were reduced using the Hectospec package. The relative flux calibration was done observing the standard star Hiltm600 (Massey et al. 1988) during the nights of October 15 and November 27. The emission-line fluxes were measured with the package SPLOT of IRAF $^{1}$. Errors in the

\footnotetext{
1 IRAF is distributed by the National Optical Astronomy Observatory, which is operated by the Association of Universities for Research in
} 
L. Magrini et al.: Metal production in M 33

Table 1. MMT observations of $\mathrm{H}$ II regions with chemical abundance determination.

\begin{tabular}{llcc}
\hline \hline $\begin{array}{l}\text { New sample } \\
(1)\end{array}$ & ID & $\begin{array}{c}\text { RA } \\
(3)\end{array}$ & $\begin{array}{c}\text { Dec } \\
(4)\end{array}$ \\
\hline 1 & BCLMP 275A & $1: 32: 29.5$ & $30: 36: 07.90$ \\
2 & GDK99 3 & $1: 32: 31.7$ & $30: 35: 27.39$ \\
3 & LGCHII14 & $1: 32: 33.3$ & $30: 32: 01.90$ \\
4 & CPSDP 26 & $1: 32: 33.7$ & $30: 27: 06.60$ \\
5 & LGCHII15 & $1: 32: 40.8$ & $30: 24: 24.99$ \\
6 & EPR2003 87 & $1: 32: 42.4$ & $30: 22: 25.59$ \\
7 & LGCHII16 & $1: 32: 43.5$ & $30: 35: 17.29$ \\
8 & LGCHII17 & $1: 33: 11.3$ & $30: 39: 03.39$ \\
9 & BCLMP 694 & $1: 33: 52.1$ & $30: 47: 15.59$ \\
10 & BCLMP 759 & $1: 33: 56.8$ & $30: 22: 16.50$ \\
11 & MJ98 WR 112 & $1: 33: 57.3$ & $30: 35: 11.09$ \\
12 & LGCHII18 & $1: 33: 58.9$ & $30: 55: 31.30$ \\
13 & BCLMP 282 & $1: 32: 39.1$ & $30: 40: 42.10$ \\
14 & BCLMP 264 & $1: 32: 40.2$ & $30: 22: 34.70$ \\
15 & BCLMP 238 & $1: 32: 44.5$ & $30: 34: 54.30$ \\
16 & BCLMP 239 & $1: 32: 51.8$ & $30: 33: 05.20$ \\
17 & BCLMP 261 & $1: 32: 54.1$ & $30: 23: 18.70$ \\
18 & CPSDP 123 & $1: 33: 20.4$ & $30: 32: 49.20$ \\
19 & CPSDP 43A & $1: 33: 23.9$ & $30: 26: 15.00$ \\
\hline Control sample & & & \\
20 & LGCHII2 & $1: 32: 43.0$ & $30: 19: 31.19$ \\
21 & LGCHII3 & $1: 32: 45.9$ & $30: 41: 35.50$ \\
22 & BCLMP289 & $1: 32: 58.5$ & $30: 44: 28.60$ \\
23 & BCLMP218 & $1: 33: 00.3$ & $30: 30: 47.30$ \\
24 & MA1 & $1: 33: 03.4$ & $30: 11: 18.70$ \\
25 & BCLMP290 & $1: 33: 11.4$ & $30: 45: 15.09$ \\
26 & IC 132 & $1: 33: 15.8$ & $30: 56: 45.00$ \\
27 & BCLMP45 & $1: 33: 29.0$ & $30: 40: 24.79$ \\
28 & BCLMP670 & $1: 34: 03.3$ & $30: 53: 09.29$ \\
29 & MA2 & $1: 34: 15.5$ & $30: 37: 11.00$ \\
30 & BCLMP691 & $1: 34: 16.6$ & $30: 51: 53.99$ \\
31 & IC 131 & $1: 33: 15.0$ & $30: 45: 09.00$ \\
32 & IC 133 & $1: 33: 15.9$ & $30: 53: 01.00$ \\
33 & BCLMP745 & $1: 34: 37.6$ & $30: 34: 55.00$ \\
\hline & & & \\
& & & \\
& & &
\end{tabular}

fluxes were calculated taking the statistical error in the measurement of the fluxes into account, as well as systematic errors of the flux calibrations, background determination, and sky subtraction. The observed line fluxes were corrected for the effect of the interstellar extinction using the extinction law of Mathis (1990) with $R_{V}=3.1$. We derived $c(\mathrm{H} \beta)$, the logarithmic nebular extinction, by using the weighted average of the observed-totheoretical Balmer ratios of $\mathrm{H} \alpha, \mathrm{H} \gamma$, and $\mathrm{H} \delta$ to $\mathrm{H} \beta$ (Osterbrock $\&$ Ferland 1989). The detailed description of the data reduction and the plasma and chemical analysis can be found in Magrini et al. (2009a, hereafter M09). Spectra of two H II regions, one close to the galactic centre and one in the outer part of the disk are shown in Fig. 1.

Table 2 gives the results of our emission-line measurements and extinction corrections for $33 \mathrm{H}$ II regions whose spectra were suitable for determining physical and chemical properties. The columns of Table 2 indicate: (1) H II region name; (2) nebular extinction coefficient $c(\mathrm{H} \beta)$ with its error; (3) emitting ion; (4) rest-frame wavelength in $\AA$; (5) measuredline fluxes;

Astronomy (AURA) under cooperative agreement with the National Science Foundation.

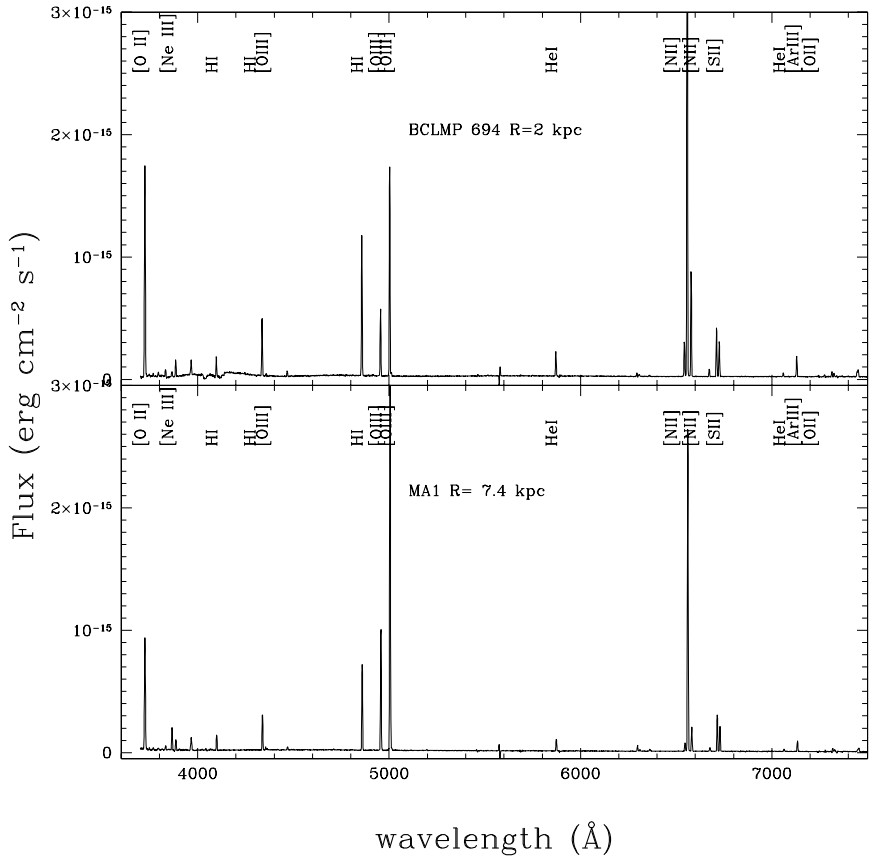

Fig. 1. Two example spectra of $\mathrm{H}$ II regions located at different galactocentric distance: BCLMP 694 at $\sim 2 \mathrm{kpc}$ and MA1 at $\sim 7.4 \mathrm{kpc}$.

(6) absolute errors on the measured line fluxes; (7) extinction corrected line fluxes. Both $F_{\lambda}(5)$ and $I_{\lambda}$ (7) are expressed on a scale where $\mathrm{H} \beta=100$. Table 2 is published in its entirety at the CDS. A portion is shown here for guidance regarding its form and content. The analysed $\mathrm{H}$ II regions represent about $2 / 3$ of our sample. The remaining $1 / 3 \mathrm{H}$ II regions have noisy spectra and are distributed at all galactocentric radii. We used the extinction-corrected intensities to obtain the electron densities and temperatures. Electronic density was derived from the intensities of the sulphur-line doublet [S II] 6716, $6731 \AA$. We used the intensities of several emission-line ratios, when available, to derive low and medium-excitation temperatures (see also Osterbrock \& Ferland 1989, Sect. 5.2): $[\mathrm{N}$ II $] \lambda 5755 /(\lambda 6548+\lambda 6584)$ and $[\mathrm{O}$ II $] \lambda 3727 /(\lambda 7320+\lambda 7330)$ for low-excitation $T_{\mathrm{e}}$, while $[\mathrm{O} \mathrm{III}] \lambda 4363 /(\lambda 5007+\lambda 4959)$ and $[\mathrm{S}$ III $] \lambda 6312 /(\lambda 9069+\lambda 9532)$ for medium-excitation $T_{\mathrm{e}}$. We performed plasma diagnostics and ionic abundance calculation by using the 5-level atom model included in the nebular analysis package in IRAF/STSDAS (Shaw \& Dufour 1994). The elemental abundances are then determined by applying the ionization correction factors (ICFs) following the prescriptions by Kingsburgh \& Barlow (1994) for the case where only optical lines are available. In the abundance analysis we adopted $T_{\mathrm{e}}[\mathrm{N} \mathrm{II}]$ and/or $T_{\mathrm{e}}[\mathrm{O} \mathrm{II}]$ for computing the $\mathrm{N}^{+}, \mathrm{O}^{+}, \mathrm{S}^{+}$abundances, while $T_{\mathrm{e}}[\mathrm{O} \mathrm{III}]$ and/or $T_{\mathrm{e}}[\mathrm{S} \mathrm{III}]$ for $\mathrm{O}^{2+}, \mathrm{S}^{2+}, \mathrm{Ar}^{2+}, \mathrm{He}^{+}$, and $\mathrm{He}^{2+}$. We calculated the abundances of He I and He II using the equations of Benjamin et al. (1999) in two density regimes, i.e. $n_{\mathrm{e}}>1000 \mathrm{~cm}^{-3}$ and $\leq 1000 \mathrm{~cm}^{-3}$. The Clegg's collisional populations were taken into account (Clegg 1987). In Table 3 we present the electron densities and temperatures, and the ionic and total chemical abundances of our H II region sample, which only includes $\mathrm{H}$ II regions with at least one measured value of $T_{\mathrm{e}}$. The columns of Table 3 present: (1) identification name; (2) label of each plasma diagnostic and abundances available; (3) relative values obtained from our analysis. Table 3 is published in its entirety at the CDS.

We derived the temperature and density uncertainties using the error propagation of the absolute errors on the line fluxes. 
Table 2. Observed and de-reddened fluxes (full table available at the CDS).

\begin{tabular}{|c|c|c|c|c|c|c|}
\hline $\begin{array}{l}\text { ID } \\
(1)\end{array}$ & $\begin{array}{l}c(\mathrm{H} \beta) \\
(2)\end{array}$ & $\begin{array}{l}\text { Ion } \\
\text { (3) }\end{array}$ & $\begin{array}{l}\lambda(\AA) \\
(4)\end{array}$ & $\begin{array}{l}F_{\lambda} \\
(5)\end{array}$ & $\begin{array}{l}\Delta\left(F_{\lambda}\right) \\
(6)\end{array}$ & $\begin{array}{l}I_{\lambda} \\
(7)\end{array}$ \\
\hline \multirow[t]{19}{*}{ BCLMP275A } & $0.493 \pm 0.009$ & [OII] & 3727 & 186.4 & 2.2 & 256.7 \\
\hline & & {$[\mathrm{NeIII}] / \mathrm{HI}$} & 3968 & 3.2 & 0.7 & 4.1 \\
\hline & & HI & 4100 & 13.0 & 1.1 & 16.2 \\
\hline & & HI & 4340 & 34.4 & 1.1 & 39.8 \\
\hline & & [OIII $]$ & 4363 & 1.1 & 0.8 & 1.2 \\
\hline & & HI & 4861 & 100.0 & 1.6 & 100.1 \\
\hline & & [OIII] & 4959 & 57.3 & 1.2 & 55.8 \\
\hline & & [OIII] & 5007 & 169.9 & 2.0 & 163.3 \\
\hline & & HeI & 5876 & 13.5 & 0.9 & 10.7 \\
\hline & & [SIII] & 6312 & 1.7 & 0.8 & 1.3 \\
\hline & & {$[\mathrm{NII}]$} & 6548 & 9.1 & 1.0 & 6.5 \\
\hline & & HI & 6563 & 409.6 & 2.3 & 290.2 \\
\hline & & {$[\mathrm{NII}]$} & 6584 & 28.9 & 1.1 & 20.4 \\
\hline & & $\mathrm{HeI}$ & 6678 & 3.9 & 0.8 & 2.7 \\
\hline & & {$[\mathrm{SII}]$} & 6717 & 32.7 & 1.1 & 22.6 \\
\hline & & [SII] & 6731 & 23.1 & 1.2 & 15.9 \\
\hline & & $\mathrm{HeI}$ & 7065 & 2.6 & 0.8 & 1.7 \\
\hline & & [ArIII] & 7135 & 9.8 & 0.8 & 6.4 \\
\hline & & [SIII] & 9069 & 21.4 & 1.0 & 10.2 \\
\hline \multirow[t]{22}{*}{ GDK99 3} & $0.448 \pm 0.007$ & [OII] & 3727 & 83.3 & 1.0 & 111.4 \\
\hline & & HI & 3835 & 6.8 & 0.6 & 8.8 \\
\hline & & [NeIII] & 3869 & 25.9 & 0.6 & 33.4 \\
\hline & & $\mathrm{HeI}$ & 3889 & 9.8 & 0.6 & 12.6 \\
\hline & & {$[\mathrm{NeIII}] / \mathrm{HI}$} & 3968 & 18.0 & 0.7 & 22.7 \\
\hline & & HI & 4100 & 17.5 & 0.7 & 21.2 \\
\hline & & $\mathrm{HI}$ & 4340 & 40.0 & 0.9 & 45.8 \\
\hline & & [OIII] & 4363 & 3.3 & 0.5 & 3.8 \\
\hline & & HeI & 4471 & 3.5 & 0.5 & 3.8 \\
\hline & & HeII & 4686 & 0.5 & 0.5 & 0.6 \\
\hline & & HI & 4861 & 100.0 & 1.3 & 100.1 \\
\hline & & [OIII] & 4959 & 196.2 & 1.5 & 191.5 \\
\hline & & [OIII $]$ & 5007 & 585.9 & 2.8 & 565.0 \\
\hline & & HeI & 5876 & 14.0 & 0.7 & 11.3 \\
\hline & & [NII] & 6548 & 6.1 & 0.4 & 4.5 \\
\hline & & $\mathrm{HI}$ & 6563 & 396.4 & 1.9 & 289.9 \\
\hline & & [NII] & 6584 & 19.2 & 0.6 & 14.0 \\
\hline & & $\mathrm{HeI}$ & 6678 & 4.3 & 0.4 & 3.1 \\
\hline & & [SII] & 6717 & 31.0 & 0.7 & 22.2 \\
\hline & & [SII] & 6731 & 21.6 & 0.7 & 15.4 \\
\hline & & $\mathrm{HeI}$ & 7065 & 3.4 & 0.5 & 2.3 \\
\hline & & [ArIII] & 7135 & 14.8 & 0.5 & 9.9 \\
\hline
\end{tabular}

The errors on the ionic and total abundances were computed taking the uncertainties in the observed fluxes, in the electron temperatures and densities, and in $c(\mathrm{H} \beta)$ into account. In Table 4 a summary of the total abundances $\mathrm{He} / \mathrm{H}, \mathrm{O} / \mathrm{H}, \mathrm{N} / \mathrm{H}, \mathrm{Ne} / \mathrm{H}, \mathrm{S} / \mathrm{H}$ and $\mathrm{Ar} / \mathrm{H}$ and their errors are presented. The He abundance is shown by number with its absolute error, while the metal abundances are expressed in the form of $\log (\mathrm{X} / \mathrm{H})+12$ with errors expressed in dex. The last row indicates the average abundances computed by number.

\subsection{The PN data-set}

The PN population of M 33 was studied by Magrini et al. (2009a) using multi-fiber spectroscopy with Hectospec at the MMT with the same spectroscopic setup and during the same nights as the $\mathrm{H}$ II region observations presented here. Spectra of $102 \mathrm{PNe}$ were analysed and plasma diagnostics and chemical abundances obtained for 93 PNe where the necessary diagnostic lines were measured. The data reduction and the plasma diagnostics
Table 3. Plasma diagnostics and abundances (full table available at the CDS).

\begin{tabular}{|c|c|c|}
\hline $\begin{array}{l}\text { ID } \\
(1)\end{array}$ & (2) & (3) \\
\hline BCLMP275A & $\begin{array}{l}T_{\mathrm{e}}[\mathrm{O} \mathrm{III}] \\
T_{\mathrm{e}}[\mathrm{S} \mathrm{III}] \\
\mathrm{HeI} / \mathrm{H} \\
{[\mathrm{OII}] / \mathrm{H}} \\
{[\mathrm{OIII}] / \mathrm{H}} \\
\mathrm{ICF}(\mathrm{O}) \\
\mathrm{O} / \mathrm{H} \\
{[\mathrm{NII}] / \mathrm{H}} \\
\mathrm{ICF}(\mathrm{N}) \\
\mathrm{N} / \mathrm{H} \\
{[\mathrm{ArIII}] / \mathrm{H}} \\
\mathrm{ICF}(\mathrm{Ar}) \\
\mathrm{Ar} / \mathrm{H} \\
{[\mathrm{SII}] / \mathrm{H}} \\
{[\mathrm{SIII}] / \mathrm{H}} \\
\mathrm{ICF}(\mathrm{S}) \\
\mathrm{S} / \mathrm{H}\end{array}$ & $\begin{array}{l}10600 \\
15800 \\
0.076 \\
7.740 \mathrm{e}-05 \\
4.737 \mathrm{e}-05 \\
1.000 \\
1.248 \mathrm{e}-04 \\
3.287 \mathrm{e}-06 \\
1.612 \\
5.299 \mathrm{e}-06 \\
4.910 \mathrm{e}-07 \\
1.87 \\
9.182 \mathrm{e}-07 \\
7.486 \mathrm{e}-07 \\
1.100 \mathrm{e}-06 \\
1.019 \\
1884 \mathrm{e}-06\end{array}$ \\
\hline GDK99 3 & $\begin{array}{l}T_{\mathrm{e}}[\mathrm{O} \mathrm{III}] \\
\mathrm{HeI} / \mathrm{H} \\
{[\mathrm{OII}] / \mathrm{H}} \\
{[\mathrm{OIII}] / \mathrm{H}} \\
\mathrm{ICF}(\mathrm{O}) \\
\mathrm{O} / \mathrm{H} \\
{[\mathrm{NII}] / \mathrm{H}} \\
\mathrm{ICF}(\mathrm{N}) \\
\mathrm{N} / \mathrm{H} \\
{[\mathrm{NeIII}] / \mathrm{H}} \\
\mathrm{ICF}(\mathrm{Ne}) \\
\mathrm{Ne} / \mathrm{H} \\
{[\mathrm{ArIII}] / \mathrm{H}} \\
\mathrm{ICF}(\mathrm{Ar}) \\
\mathrm{Ar} / \mathrm{H} \\
{[\mathrm{SII}] / \mathrm{H}} \\
\mathrm{ICF}(\mathrm{S}) \\
\mathrm{S} / \mathrm{H}\end{array}$ & $\begin{array}{l}10200 \\
0.081 \\
3.880 \mathrm{e}-05 \\
1.860 \mathrm{e}-04 \\
1.006 \\
2.261 \mathrm{e}-04 \\
2.536 \mathrm{e}-06 \\
5.826 \\
1.475 \mathrm{e}-05 \\
3.150 \mathrm{e}-05 \\
1.215 \\
3.829 \mathrm{e}-05 \\
8.560 \mathrm{e}-07 \\
1.87 \\
1.601 \mathrm{e}-06 \\
8.157 \mathrm{e}-07 \\
1.323 \\
8.255 \mathrm{e}-06\end{array}$ \\
\hline
\end{tabular}

followed exactly the same procedure as described in the present paper, thus ensuring that no biases are introduced for the different analysis of the spectra. About $20 \%$ of the studied PNe have young progenitors, the so-called type I PNe. The rest of the PNe in the sample are the progenies of an old disk stellar population, with main sequence masses $M<3 M_{\odot}$ and ages $t>0.3$ Gyr. A tight relation between the $\mathrm{O} / \mathrm{H}$ and $\mathrm{Ne} / \mathrm{H}$ abundances was found, excluding that both elements have been altered by PN progenitors and supporting the validity of oxygen as a good tracer of the ISM composition at the epoch of the progenitors' birth.

\section{The metallicity distribution in M 33}

The large amount of chemical abundance data from $\mathrm{H}$ II regions in M 33 allow us to analyse the spatially-resolved distribution of metals in the ISM. In this section, we present the radial distribution and the map of $\mathrm{O} / \mathrm{H}$, using the new data presented in this paper and all previous oxygen determinations for which $T_{\mathrm{e}}$ has been measured. 
Table 4. The chemical abundances of our MMT sample.

\begin{tabular}{|c|c|c|c|c|c|c|}
\hline $\begin{array}{l}\# \\
\text { (1) }\end{array}$ & $\begin{array}{l}\mathrm{He} / \mathrm{H} \\
\text { (2) }\end{array}$ & $\begin{array}{l}\mathrm{O} / \mathrm{H} \\
(3)\end{array}$ & $\begin{array}{l}\mathrm{N} / \mathrm{H} \\
(4)\end{array}$ & $\begin{array}{l}\mathrm{Ne} / \mathrm{H} \\
(5)\end{array}$ & $\begin{array}{l}\mathrm{Ar} / \mathrm{H} \\
(6)\end{array}$ & $\begin{array}{l}S / H \\
\text { (7) }\end{array}$ \\
\hline 1 & $0.076 \pm 0.001$ & $8.10 \pm 0.07$ & $6.72 \pm 0.15$ & - & $5.96 \pm 0.34$ & $6.27 \pm 0.20$ \\
\hline 2 & $0.081 \pm 0.001$ & $8.35 \pm 0.05$ & $7.17 \pm 0.11$ & $7.58 \pm 0.015$ & $6.20 \pm 0.09$ & $6.92 \pm 0.21$ \\
\hline 3 & $0.058 \pm 0.003$ & $7.97 \pm 0.08$ & $6.88 \pm 0.12$ & - & $6.02 \pm 0.11$ & $6.62 \pm 0.15$ \\
\hline 4 & $0.088 \pm 0.010$ & $8.36 \pm 0.05$ & $7.24 \pm 0.19$ & - & $5.82 \pm 0.18$ & $6.46 \pm 0.19$ \\
\hline 5 & $0.085 \pm 0.005$ & $8.45 \pm 0.02$ & $7.35 \pm 0.14$ & - & - & $6.59 \pm 0.12$ \\
\hline 6 & $0.114 \pm 0.003$ & $8.52 \pm 0.08$ & $6.86 \pm 0.15$ & $7.60 \pm 0.20$ & $6.27 \pm 0.12$ & $6.50 \pm 0.20$ \\
\hline 7 & $0.085 \pm 0.005$ & $8.30 \pm 0.12$ & $7.09 \pm 0.20$ & $7.00 \pm 0.1$ & $6.07 \pm 0.16$ & $6.61 \pm 0.15$ \\
\hline 8 & $113 \pm 0.005$ & $8.21 \pm 0.07$ & $7.30 \pm 0.24$ & $7.69 \pm$ & $6.20 \pm 0.20$ & $6.49 \pm 0.15$ \\
\hline 9 & $092 \pm 0$ & $3 \pm 0.10$ & $7.21 \pm 0.10$ & 7.5 & $6.22 \pm 0.30$ & $6.79 \pm 0.10$ \\
\hline 10 & $0.077 \pm($ & $8.11 \pm$ & $6.88 \pm 0$ & - & \pm 0.22 & $6.66 \pm 0.15$ \\
\hline 11 & $0.093 \pm 0.003$ & $8.30 \pm 0.07$ & $37 \pm 0.02$ & - & $0 \pm 0.30$ & $7.09 \pm 0.11$ \\
\hline 12 & $0.091 \pm 0.008$ & $8.15 \pm 0.07$ & $7.94 \pm 0.05$ & $7.20 \pm 0.10$ & $6.24 \pm 0.10$ & $6.51 \pm 0.20$ \\
\hline 13 & $0.080 \pm 0.005$ & $8.32 \pm 0.05$ & $6.92 \pm 0.12$ & - & $6.19 \pm 0.12$ & $6.36 \pm 0.18$ \\
\hline 14 & $0.076 \pm 0.003$ & $7.87 \pm 0.08$ & $6.93 \pm 0.15$ & $7.11 \pm 0.20$ & $5.89 \pm 0.20$ & $6.41 \pm 0.18$ \\
\hline 15 & $0.089 \pm 0.006$ & $7.92 \pm 0.08$ & $6.75 \pm 0.15$ & - & $5.92 \pm 0.18$ & $6.57 \pm 0.15$ \\
\hline 16 & $0.086 \pm 0.005$ & $8.27 \pm 0.07$ & $7.20 \pm 0.14$ & - & - & $6.52 \pm 0.20$ \\
\hline 17 & $0.115 \pm 0.008$ & $8.01 \pm 0.08$ & $6.62 \pm 0.16$ & $7.16 \pm 0.18$ & $5.93 \pm 0.14$ & $6.40 \pm 0.18$ \\
\hline 18 & $0.078 \pm 0.005$ & $8.35 \pm 0.05$ & $7.35 \pm 0.12$ & - & $6.35 \pm 0.15$ & $6.94 \pm 0.20$ \\
\hline 19 & $0.059 \pm 0.003$ & $8.40 \pm 0.05$ & $8.05 \pm 0.10$ & $8.03 \pm 0.18$ & $5.16 \pm 0.18$ & $7.02 \pm 0.17$ \\
\hline 20 & $0.083 \pm 0.001$ & $8.08 \pm 0.05$ & $6.91 \pm 0.15$ & $6.87 \pm 0.18$ & $6.18 \pm 0.25$ & $6.64 \pm 0.15$ \\
\hline 21 & $0.086 \pm 0.001$ & $8.42 \pm 0.06$ & $7.56 \pm 0.13$ & $7.31 \pm$ & \pm 0.27 & 0.10 \\
\hline 22 & \pm 0.005 & 0.12 & \pm 0.16 & $7.75 \pm$ & .50 & 0.11 \\
\hline 23 & & & & - & 0.14 & 0.23 \\
\hline 24 & $0.080 \pm 0.008$ & $8.28 \pm 0.15$ & $7.10 \pm 0.20$ & 7.61 & \pm 0.32 & $6.71 \pm 0.40$ \\
\hline 25 & $6 \pm 0.005$ & $8 \pm 0.13$ & 7.3 & & 0.10 & $6.57 \pm 0.15$ \\
\hline 26 & $0.061 \pm 0.003$ & $7.98 \pm 0.05$ & $6.98 \pm 0.15$ & $7.28=$ & $5.86 \pm 0.15$ & $6.36 \pm 0.13$ \\
\hline 27 & $0.095 \pm 0.001$ & $8.48 \pm 0.08$ & $7.62 \pm 0.12$ & $7.73 \pm 0.15$ & $6.33 \pm 0.25$ & $6.87 \pm 0.15$ \\
\hline 28 & $0.088 \pm 0.002$ & $8.30 \pm 0.07$ & $7.10 \pm 0.18$ & $7.42 \pm$ & $6.23 \pm 0.30$ & $6.63 \pm 0.20$ \\
\hline 29 & $0.091 \pm 0.005$ & $8.31 \pm 0.10$ & $7.19 \pm 0.15$ & $7.46 \pm 0.25$ & $6.42 \pm 0.24$ & $6.92 \pm 0.21$ \\
\hline 30 & $0.096 \pm 0.003$ & $8.42 \pm 0.06$ & $7.20 \pm 0.12$ & $7.85 \pm 0.21$ & $6.37 \pm 0.32$ & $6.75 \pm 0.23$ \\
\hline 31 & $0.097 \pm 0.005$ & $8.47 \pm 0.08$ & $7.26 \pm 0.15$ & $7.76 \pm 0.20$ & $6.29 \pm 0.25$ & $7.06 \pm 0.15$ \\
\hline 32 & $0.079 \pm 0.005$ & $8.27 \pm 0.08$ & $7.21 \pm 0.17$ & $7.58 \pm 0.21$ & $5.49 \pm 0.30$ & $6.83 \pm 0.15$ \\
\hline \multirow[t]{2}{*}{33} & $0.067 \pm 0.008$ & $7.93 \pm 0.10$ & $7.10 \pm 0.20$ & - & $6.09 \pm 0.21$ & $6.51 \pm 0.20$ \\
\hline & $0.085 \pm 0.011$ & $8.27_{-0.17}^{+0.12}$ & $7.31_{-0.35}^{+0.30}$ & $7.56_{-0.30}^{+0.18}$ & $6.13_{-0.22}^{+0.14}$ & $6.71_{-0.34}^{+0.19}$ \\
\hline
\end{tabular}

\subsection{The metallicity gradient of $\mathrm{H} / \mathrm{l}$ regions}

Our cumulative sample includes: i) H II regions by Magrini et al. (2007a), which includes abundances from their own sample and previous abundance determinations (all with $T_{\mathrm{e}}$ and with abundances recomputed uniformly); ii) the sample by Rosolowsky \& Simon (2008); iii) the present sample (see Table 3). In Fig. 2, we show the oxygen abundance as a function of galactocentric distance for the cumulative sample of $\mathrm{H}$ II regions. In this figure each point corresponds to a single region; i.e., we do not plot multiple measurements for the same region but only the value with the lowest error. Note the large dispersion in the radial region between 1 and $2 \mathrm{kpc}$ from the centre caused by several high- and low-metallicity regions, located in the southern arm (see Sect. 3.3), which might be related to the presence of a bar (e.g., Corbelli \& Walterbos 2007). We applied the routine fitexy in Numerical Recipes (Press et al. 1992) to fit the relation between the oxygen abundances and the galactocentric distances, taking their errors into account and minimizing $\chi^{2}$. Typical errors on the de-projected galactocentric distances associated with the uncertainty on the inclination were less than $0.1 \mathrm{kpc}$ (Magrini et al. 2007a). The fit to the complete sample gives a gradient of

$12+\log (\mathrm{O} / \mathrm{H})=-0.037( \pm 0.009) R_{\mathrm{GC}}+8.465( \pm 0.038)$

where $R_{\mathrm{GC}}$ is the de-projected galactocentric distance in $\mathrm{kpc}$, computed by assuming an inclination of $53^{\circ}$ and a position angle of $22^{\circ}$. A weighted linear least-square fit to the MMT sample only gives a gradient of

$12+\log (\mathrm{O} / \mathrm{H})=-0.044( \pm 0.017) R_{\mathrm{GC}}+8.447( \pm 0.084)$,

which is consistent within the errors with the gradient from the larger sample. In the rest of the paper, we use the larger and more complete sample when discussing the metallicity gradient and its possible time variation, but excluding the first kpc region where only a few low metallicity regions were analysed. We discuss the possible reasons for the lower value of the central metallicity later in this section and in Sect. 4. The $\mathrm{O} / \mathrm{H}$ gradient of the whole sample of $\mathrm{H}$ II regions sample, excluding the central $1 \mathrm{kpc}$, is

$12+\log (\mathrm{O} / \mathrm{H})=-0.044( \pm 0.009) R_{\mathrm{GC}}+8.498( \pm 0.041)$.

We also checked the metallicity gradient by tracing it in different areas of the galaxy, namely in the northwest and in the southeast halves, separately, and in the nearest and in the farthest sides. The results are shown in Fig. 3. The northern and southern gradients, as well as those relative to the nearest and farthest sides, are identical within the uncertainties, with slopes around $-0.03-4 \mathrm{dex} \mathrm{kpc}^{-1}$. The only difference found between the metallicity gradients obtained for sections of the galaxy is the presence of a high metallicity peak in the southern arm. In conclusion, the present H II region sample (literature + presentwork, 103 objects), including only nebulae with measured $T_{\mathrm{e}}$, 


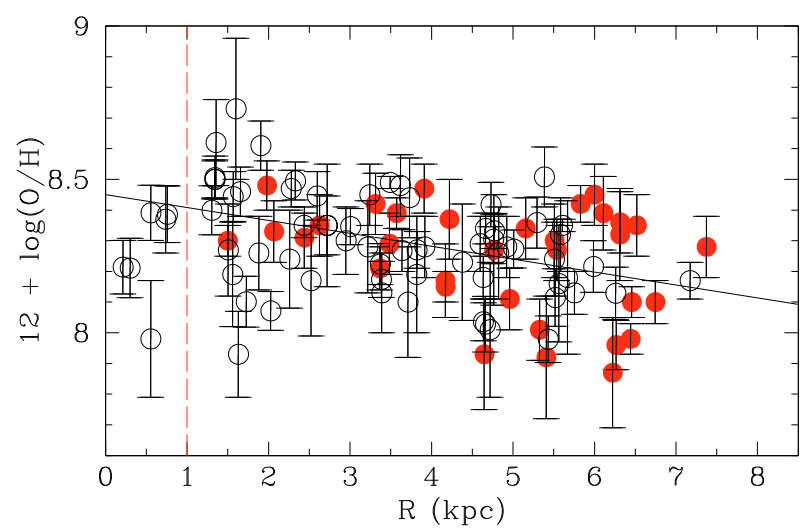

Fig. 2. The $\mathrm{O} / \mathrm{H}$ radial gradient for the cumulative $\mathrm{H}$ II region sample: filled circles are the MMT observations (new and control samples), empty circles are the literature abundances. The continuous line is the weighted linear least-square fit of Eq. (3), i.e., with a radial range from 1 to $8 \mathrm{kpc}$ from the M 33 centre. The dashed vertical line indicates the regions located at its left-side and excluded from the fit.

reinforce the recent results on the slope of the $\mathrm{M} 33 \mathrm{O} / \mathrm{H}$ gradient, with a global slope up to around $8 \mathrm{kpc}$ of $-0.03 \mathrm{dex} \mathrm{kpc}^{-1}$, and of about $-0.04 \mathrm{dex} \mathrm{kpc}^{-1}$ excluding the central $1 \mathrm{kpc}$. The very central regions remain somewhat undersampled (6 objects within $1 \mathrm{kpc}$ from the centre, and 9 within $1.5 \mathrm{kpc}$ ) and in disagreement with other results. A comparison with the metallicity gradient derived from young stars, which are representative of the same epoch in the lifetime of the galaxy as H II regions, and with the infrared spectroscopy of $\mathrm{H}$ II regions, is necessary.

Stellar abundances were obtained by Herrero et al. (1994) for AB-supergiants, McCarthy et al. (1995) and Venn et al. (1998) for A-type supergiant stars, and Monteverde et al. (1997, 2000) and Urbaneja et al. (2005) for B-type supergiant stars. The largest sample of Urbaneja et al. (2005) gave a $\mathrm{O} / \mathrm{H}$ gradient of $-0.06 \pm 0.02 \mathrm{dex} \mathrm{kpc}^{-1}$. Recently, U et al. (2009) has presented spectroscopic observations of a set of A and B supergiants. They determined stellar metallicities and derived the metallicity gradient in the disk of M 33, finding solar metallicity at the centre and 0.3 solar in the outskirts at a distance of $8 \mathrm{kpc}$. Their average metallicity gradient is $-0.07 \pm 0.01 \mathrm{dex} \mathrm{kpc}^{-1}$. At a given radius, $\mathrm{H}$ II regions have abundances slightly below the stellar results, and this is probably due to the depletion of oxygen in $\mathrm{H}$ II regions on dust grains (e.g., Bresolin et al. 2009). The slopes of the two gradients agree if the comparison is done between about 1 ant $8 \mathrm{kpc}$. The cause of the difference between the supergiant and $\mathrm{H}$ II region gradient is the metallicity value in the central regions. In fact, the $\mathrm{H}$ II regions located within $1 \mathrm{kpc}$ from centre have metallicity below solar, whereas the supergiants are metal rich, ranging from solar values to above solar. The origin of this discrepancy is not the temperature gradients within the nebulae (Stasińska 2005) because they become important at higher metal abundances.

Recent observations of $25 \mathrm{H}$ II regions by Rubin et al. (2008) with Spitzer have allowed a measurement of the $\mathrm{Ne} / \mathrm{H}$ and $\mathrm{S} / \mathrm{H}$ gradient across the disk of M 33 showing no decrease in chemical abundances in the central regions. Infrared $\mathrm{Ne}$ and $\mathrm{S}$ emission lines do not have a strong dependence on $T_{\mathrm{e}}$, and consequently their abundances can be determined even without a temperature measurement.

One way to explain the low metallicity in the central $1.0 \times$ $1.0 \mathrm{kpc}^{2}$ area is related to the criterion used for $\mathrm{H}$ II regions. Usually, chemical abundances derived from optical spectroscopy
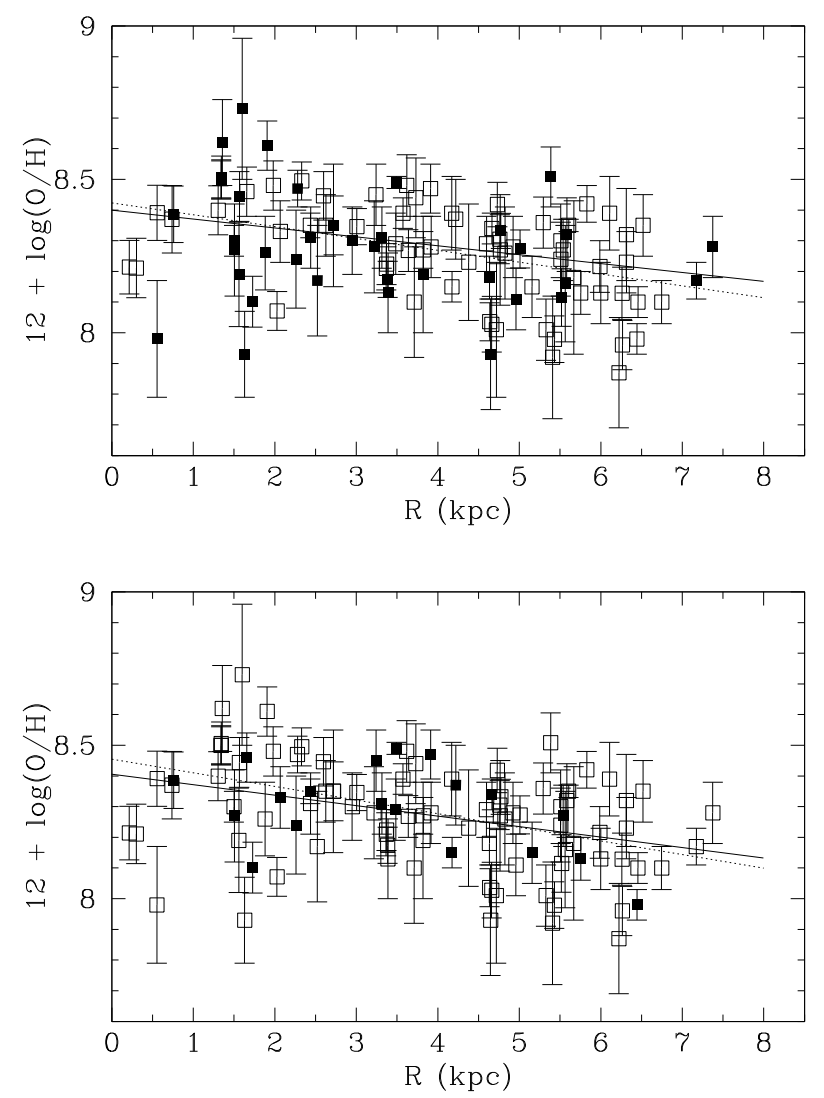

Fig. 3. The $\mathrm{O} / \mathrm{H}$ radial gradient obtained in different regions of $\mathrm{M} 33$. Top: nearest side (filled squares) and farthest side (empty squares) gradient. Bottom: North (filled squares) and South (empty squares) gradient. In each panel the weighted linear least-square fits of the two regions are shown with two lines (continuous and dotted).

rely on direct measurement of the electron temperature, given by the [O III] $4363 \AA$ emission line. This emission line is inversely proportional to the oxygen abundance and barely detectable for $\mathrm{O} / \mathrm{H}>8.6$ from an average luminosity nebula (e.g., Nagao et al. 2006). The request for [O III] $4363 \AA$ Adetection might determine a bias towards lower metallicity, with the exclusion of the highest metallicity nebulae. This could explain the differences between the optical spectroscopy results and both the stellar abundance determinations, and the $\mathrm{H}$ II region infrared spectroscopy.

\subsection{The abundance gradients of the other chemical elements}

Our analysis allowed us to measure other chemical elements in addition to oxygen, as $\mathrm{He} / \mathrm{H}, \mathrm{N} / \mathrm{H}, \mathrm{Ne} / \mathrm{H}, \mathrm{S} / \mathrm{H}$ and $\mathrm{Ar} / \mathrm{H}$. The more reliable measurement is that of oxygen for the reasons illustrated in the Appendix, and we use it to follow the chemical evolution of M 33. Nevertheless the other chemical elements are measured in enough $\mathrm{H}$ II regions to compute their radial gradients.

In Table 5 we show the slopes and central abundance values of the radial gradients of $\mathrm{N} / \mathrm{H}, \mathrm{Ne} / \mathrm{H}, \mathrm{S} / \mathrm{H}$ and $\mathrm{Ar} / \mathrm{H}$ of our sample of $\mathrm{H}$ II regions. We did not calculate the radial gradient of $\mathrm{He} / \mathrm{H}$ because we measured only the ionized fraction of $\mathrm{He}$ in $\mathrm{H}$ II regions, which is only a small part of the total helium abundance. All gradients have a negative slope, consistent, within the errors, with the slope found for $\mathrm{O} / \mathrm{H}$, while $\mathrm{N} / \mathrm{H}$ is a bit steeper, as already noticed, e.g., by Magrini et al. (2007a). 
Table 5. The radial gradients of $\mathrm{N} / \mathrm{H}, \mathrm{Ne} / \mathrm{H}, \mathrm{S} / \mathrm{H}$ and $\mathrm{Ar} / \mathrm{H}$.

\begin{tabular}{lll}
\hline \hline $12+\log (\mathrm{X} / \mathrm{H})$ & slope & central value \\
$(1)$ & $(2)$ & $(3)$ \\
\hline $\mathrm{N} / \mathrm{H}$ & $-0.08 \pm 0.03$ & $7.53 \pm 0.15$ \\
$\mathrm{Ne} / \mathrm{H}$ & $-0.05 \pm 0.04$ & $7.71 \pm 0.21$ \\
$\mathrm{~S} / \mathrm{H}$ & $-0.06 \pm 0.02$ & $6.41 \pm 0.11$ \\
$\mathrm{Ar} / \mathrm{H}$ & $-0.07 \pm 0.03$ & $6.98 \pm 0.13$ \\
\hline
\end{tabular}

Its different behaviour with respect to the $\alpha$-elements, as oxygen, neon, sulphur, and argon, comes from the different places of production. $\alpha$-elements are indeed produced by SNe II, which are the final phase of the evolution of massive stars, while nitrogen is one of the final products of the evolution of long-lived low- and intermediate-mass stars. This is discussed in detail in Sect. 5. Finally, there is a very good agreement of the $\mathrm{S} / \mathrm{H}$ and $\mathrm{Ne} / \mathrm{H}$ gradients with those derived from the infrared spectra of $\mathrm{H}$ II regions by Rubin et al. (2008), for which they found a gradient of $-0.058 \pm 0.014 \mathrm{dex} \mathrm{kpc}^{-1}$ for $\mathrm{Ne} / \mathrm{H}$ and $-0.052 \pm$ $0.021 \mathrm{dex} \mathrm{kpc}^{-1}$ for $\mathrm{S} / \mathrm{H}$.

\subsection{The population-dependent metallicity gradient: giant vs. faint and compact $\mathrm{H}$ II regions}

We now examine whether any selection effect can be responsible for the difference between the steep gradient found in the early studies and the shallower gradient of this work. To this goal, we subdivided the sample of $\mathrm{H}$ II regions according to their projected size and surface brightness in the $\mathrm{H} \alpha$ emission-line. Then, we computed the intrinsic luminosity and the radius in an $\mathrm{H} \alpha$ emission-line calibrated map (courtesy of Walterbos) for each nebula of the whole sample. Defining the surface brightness (SB) as the ratio between the total flux and the area expressed in $\operatorname{arcsec}^{2}$, we subdivided the sample according to their size and SB. Considering their size, we defined them as small if their radius $R<15^{\prime \prime}$ (60 pc at the distance $840 \mathrm{kpc}$ ) and as large if $R \geq 15^{\prime \prime}$. Considering their surface brightness, we define them as bright if their surface brightness $\mathrm{SB}>5.5 \times$ $10^{-19} \mathrm{erg} \mathrm{cm}^{-2} \mathrm{~s}^{-1} \operatorname{arcsec}^{-1}$, and as faint if SB is lower than this limit. The four combinations are allowed, i.e. H II regions can be small and either bright or faint, or large and again bright or faint. In Table 6 we show the galactocentric distance, $R_{\mathrm{GC}}$, the $\mathrm{H} \alpha$ observed total flux, $F_{\mathrm{H} \alpha}$, the radius, $R$, and the $\mathrm{SB}$, of the so-called giant regions, i.e. those with $\mathrm{SB}>5.5 \times$ $10^{-19} \mathrm{erg} \mathrm{cm}^{-2} \mathrm{~s}^{-1} \operatorname{arcsec}^{-1}$ and $R \geq 15^{\prime \prime}$.

To compare the total population with the giant $\mathrm{H}$ II regions, we show in Fig. 4 the oxygen abundances of the cumulative sample, averaged in bins of $1 \mathrm{kpc}$ each, together with the abundances of each single giant $\mathrm{H}$ II region. The $\mathrm{O} / \mathrm{H}$ gradient of the $\mathrm{H}$ II regions in Table 6, computed with a weighted linear leastsquare fit, is

$12+\log (\mathrm{O} / \mathrm{H})=-0.089( \pm 0.023) R_{\mathrm{GC}}+8.72( \pm 0.09)$.

The gradient of the remaining sample is the same of given in Eq. (1). The giant regions show a significantly steeper gradient, consistent with the gradients by Smith (1975), Kwitter \& Aller (1981), Vílchez et al. (1988), and Garnett et al. (1997). The question is whether this gradient is really significantly different from the whole sample, and if this is the case, what is the reason of such different behaviour.

Owing to the small number of giant $\mathrm{H}$ II regions (9 in total), the uncertainty on the slope of their gradient is high. Thus it could still be in partial agreement, within the errors, with the
Table 6. Giant $\mathrm{H}$ II regions with derived chemical abundances.

\begin{tabular}{lllll}
\hline \hline Id & $\begin{array}{l}R_{\mathrm{GC}} \\
(\mathrm{kpc})\end{array}$ & $\begin{array}{l}F_{\mathrm{H} \alpha} \\
\left(10^{-15} \mathrm{erg} / \mathrm{cm}^{2} \mathrm{~s}\right)\end{array}$ & $\begin{array}{l}R \\
(\operatorname{arcsec})\end{array}$ & $\begin{array}{l}\mathrm{SB} \\
\left(10^{-19} \mathrm{erg} / \mathrm{cm}^{2} \mathrm{~s} \mathrm{arcsec}^{2}\right)\end{array}$ \\
\hline NGC 595 & 1.7 & 13.0 & 25 & 16.1 \\
C001Ab & 1.9 & 3.2 & 20 & 6.2 \\
MA2 & 2.5 & 4.5 & 25 & 5.6 \\
BCLMP691 & 3.3 & 2.4 & 15 & 8.2 \\
NGC 604 & 3.5 & 36.6 & 30 & 31.4 \\
IC 131 & 3.9 & 1.9 & 15 & 6.6 \\
BCLMP290 & 4.2 & 2.2 & 15 & 7.5 \\
NGC 588 & 5.5 & 4.2 & 20 & 8.0 \\
IC 132 & 6.4 & 3.5 & 20 & 6.8 \\
\hline
\end{tabular}

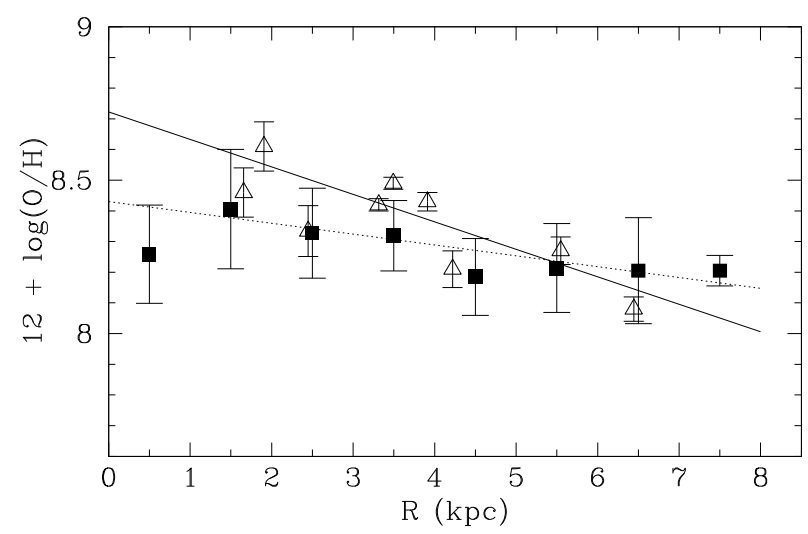

Fig. 4. The $\mathrm{O} / \mathrm{H}$ radial gradient: the giant $\mathrm{H}$ II regions (empty triangles) and the complete sample averaged in bins, each $1 \mathrm{kpc}$ wide (filled squares). The continuous line is the weighted mean least square fit of the giant $\mathrm{H}$ II region sample, while the dotted line refers to the complete sample.

larger sample, and their difference might stem from metallicity fluctuations in the ISM. On the other hand, the characteristics of the giant regions might be truly different from the average sample. For example large self-bound units are not destroyed by massive stars and thus retain their original structure and get continuously enriched by SF. However, while this might be the case for the metallicity peak near the centre, it does predict that giant regions should have higher metallicity at all galactocentric radii, which is clearly not the case. Moreover, in a recent paper, Relaño \& Kennicutt (2009) studied the star formation in luminous $\mathrm{H}$ II regions in M 33, which correspond mostly to our giant $\mathrm{H}$ II regions. They found that the observed UV and $\mathrm{H} \alpha$ luminosities are consistent with a young stellar population (3-4 Myr), born in an instantaneous burst. Thus the steeper gradient might result form a combination of a small statistics and of a metal self-enrichment effect in the giant region sample.

\subsection{The 2-dimensional distribution of metals}

The usual way to study the metallicity distribution in disk galaxies is to average it azimuthally, assuming that i) the centre of the galaxy coincides with the peak of the metallicity distribution and ii) the metallicity distribution is axially symmetric. The large number of metallicity measurements in M 33, both from $\mathrm{H}$ II regions and from PNe, allowed us to reconstruct not only their radial gradient, but also their spatial distribution projected onto the disk. In Fig. 5, we show the two-dimensional metallicity distributions for M 33 from $\mathrm{H}$ II regions and from PNe superimposed to a contour map of the stellar mass distribution derived 

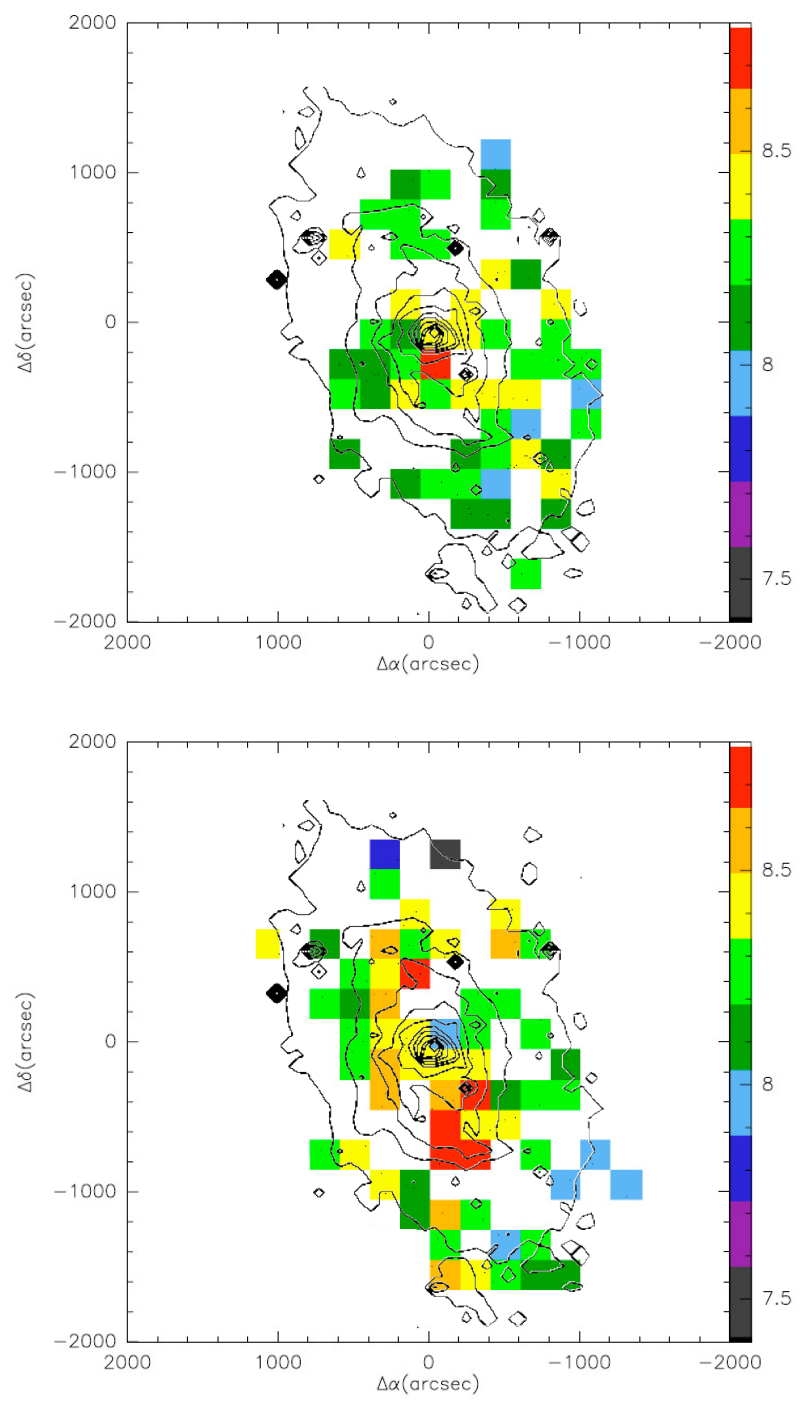

Fig. 5. The oxygen abundance maps of M $33\left(60^{\prime} \times 60^{\prime}\right)$ : H II regions (top) and $\mathrm{PNe}$ (bottom). The $\mathrm{O} / \mathrm{H}$ abundances are averaged in bins of $0.8 \times 0.8 \mathrm{kpc}^{2}$. The colour-scale shows the oxygen abundance as indicated in the label. North at the top, east to the left. The optical centre of M 33 is located at $(0,0)$. The contour levels represent the stellar mass distribution derived from the JHK image of M 33.

from the JHK image, a composition of the image of Regan \& Vogel (1994) and the 2MASS image. The O/H abundances were averaged in bins of $0.8 \times 0.8 \mathrm{kpc}^{2}$. The white pixels indicate areas where metallicity measurements are lacking: for $\mathrm{H}$ II regions they generally correspond to the interarm regions, while for PNe to spiral arms.

The H II regions with the highest metallicity are not located in the optical centre of the galaxy $(0,0$ in the map), but rather lie at radius $1-2 \mathrm{kpc}$ in the southeast direction. Also in the case of $\mathrm{PNe}$, most of the metal rich $\mathrm{PNe}$ are located in the southern part of M 33, from 2 to $4 \mathrm{kpc}$ from the centre. However the lack of known PNe in the northern spiral arm at the same distance of the southern metal-rich PNe (because of the extended H II regions not allowing the identification of stellar emission-line sources) does not allow a complete 2D picture of their metal distribution around the central regions.

To estimate the location of the off-centre metallicity peak for the $\mathrm{H}$ II region map, we divided its squared $10^{\prime} \times 10^{\prime}$ region, centered at RA 1:33:50.9 dec 30:39:36 (M 33 centre from the 2MASS survey, Skrutskie et al. 2006), with a $10 \times 10$ grid. We

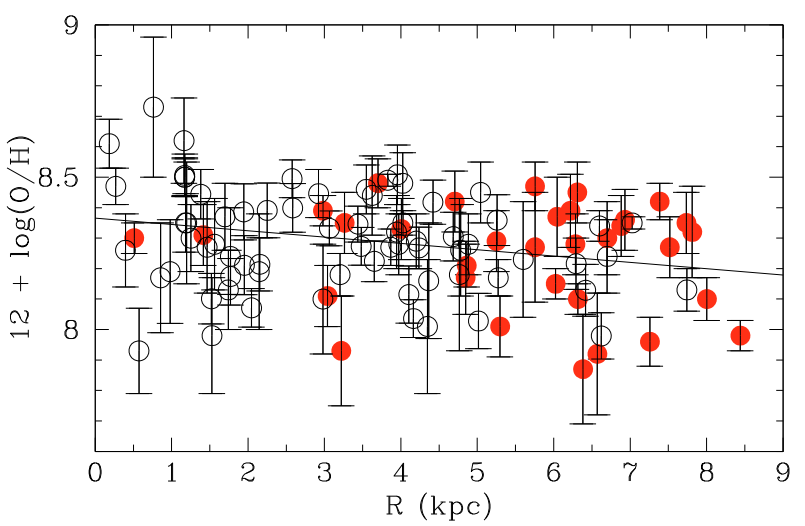

Fig. 6. The $\mathrm{O} / \mathrm{H}$ radial gradient computed with the centre located at RA 1:33:59 dec 30:33:35 (J2000.0) to minimize the dispersion in the slope of the radial gradient.

computed the radial $\mathrm{O} / \mathrm{H}$ gradients varying the central position in the grid and then finding the one that minimizes the scatter of the gradient. We found an off-centre position at RA 1:33:59 $\operatorname{dec}$ 30:33:35 (J2000.0), which corresponds to the location of the high-metallicity $\mathrm{H}$ II regions in the southern arm. The oxygen gradient measured from this central position of the whole $\mathrm{H}$ II region population, including also the central objects, is

$12+\log (\mathrm{O} / \mathrm{H})=-0.021( \pm 0.007) R_{\mathrm{GC}}+8.36( \pm 0.03)$

and it is shown in Fig. 6. The gradient of Eq. (5) is flatter and with a somewhat lower absolute dispersion than the one in Eq. (1). The reduction of the dispersion in the $\mathrm{O} / \mathrm{H}$ gradient due to the displacement of the galaxy centre is not enough strong to confirm that the metallicity maximum corresponds to the real centre of the metallicity distribution.

\section{Why is the metallicity peak off-centre?}

In the following, we examine several possibilities to explain the presence of the off-centre metallicity maximum and the low metallicity measured in the central region: i) a local effect of ISM metallicity fluctuations; ii) the lack of dominant gravitational centre in the galaxy; iii) the selection criterion of $\mathrm{H}$ II regions for metallicity determination.

\subsection{Local metallicity fluctuations}

Simon \& Rosolowsky (2008) have already noticed the nonaxisymmetric distribution of $\mathrm{H}$ II region abundances and suggest that the material enriched by the most recent generation of star formation in the arm might not have been azimuthally mixed through the galaxy. A strong OB association located in the southern arm might be responsible for the enhancement enrichment at the location of the metallicity peak. Velocity shear is present in M 33 even at small radii because of the slow rise in the rotation curve (Corbelli 2003). At the peak location, differential rotation will shear up the bubble of metals produced in the surrounding ISM in about $10^{8}$ yrs. The timescale seems long enough to allow vigorous star formation at a particular location to enrich the ISM of metals well above the average value. However, the large dispersion in the metallicity around the peak location seems to rule out an inefficient azimuthal mixing or redistribution of the metals. 


\subsection{The lack of a gravitational centre}

The non-axysymmetric metallicity distribution might be related to a general non-axisymmetric character of central regions of M 33, noticed in the past by several authors. Colin \& Athanassoula (1981) found that the young population displacement is located towards the southern side of M 33 and amounts to approximately $2-3^{\prime}$, i.e. $480-720 \mathrm{kpc}$. Using evidence of other asymmetries in the inner regions of M 33, such as those present in the distribution of $\mathrm{H} \mathrm{I}$ atomic gas, of $\mathrm{H}$ II regions, and in the kinematics, they proposed a bulge centre presently located in the northern part of the galaxy, which is rotating retrogradely around the barycenter of the galaxy. The analysis of infrared images (Minniti et al. 1993), however, seems to point out to a small bulge with a much smaller displacement the one advocated by Colin \& Athanassoula (1981). A detailed analysis of the kinematics of the innermost regions of M 33 by Corbelli \& Walterbos (2007) confirms asymmetries in the stellar and gas velocities, which however seem more related to the presence of a weak bar. The exact galaxy centre is uncertain on scales of a few arcsec. Thus, even if M 33 lacks of a dominant gravitational centre of M 33 and the bright central cluster might migrate around it, it seems unlikely that the centre of the galaxy is off by several hundreds pc from where the bright cluster lie. The marginal gain in the dispersion in re-computing the metallicity gradient from an off-centre position (see previous section) confirms that this hypothesis seems unlikely.

\subsection{Selection criterion}

That the average metallicity at the centre seems lower than at $1.5 \mathrm{kpc}$ is hard to explain in the framework of an inside-out disk formation scenario. We now discuss the possibility than in the central regions the metallicity might be higher than reported in this paper because of a bias in the H II region selection. As explained in Sect. 3.1 the inclusion of $\mathrm{H}$ II regions in our sample requires determining of the electron temperature through the detection of the faint oxygen auroral line. As the metallicity increases the line becomes so faint as to be detectable only in bright complexes. The centre of M 33 lacks of vigorous star-forming sites, so the most cooler, metal rich $\mathrm{H}$ II regions have the oxygen auroral line below the detection threshold. To prove that this might be the case we searched the literature for the existing $\mathrm{H}$ II region spectra inside $1.5 \mathrm{kpc}$ radius, which were not included in our database because of the undetectable [O III] 4363 line. We found $4 \mathrm{H}$ II regions in the database of Magrini et al. (2007a) for which optical spectroscopy is available but no detection of temperature diagnostic lines. Their names, coordinates, galactocentric distances, assumed electron temperatures, and oxygen abundances from M07a fluxes are shown in Table 7.

In Fig. 7 we plot the relationship between the electron temperature and the galactocentric distance for the complete $\mathrm{H}$ II region sample. The weighted mean least square fit gives a relationship between the two quantities

$T_{\mathrm{e}}=(410 \pm 80) \times R_{\mathrm{GC}}+8600 \pm 320$

where $T_{\mathrm{e}}$ is expressed in $\mathrm{K}$ and $R_{\mathrm{GC}}$ in kpc. We thus derived $T_{\mathrm{e}}$ for the $\mathrm{H}$ II regions of Table 7, adopting it in their chemical abundance calculations.

Using the intensities by M07a, complemented with upper limit measurements of the [O II] 7320-7330 ^ when not available in the original paper, we roughly estimated the oxygen abundance of the four $\mathrm{H}$ II regions located within $1.5 \mathrm{kpc}$. Their
Table 7. H II regions in the central regions without direct $T_{\mathrm{e}}$ measurement.

\begin{tabular}{llllll}
\hline \hline ID & RA & $\begin{array}{c}\text { Dec } \\
\text { J2000.0 }\end{array}$ & $\begin{array}{l}R_{\mathrm{GC}} \\
(\mathrm{kpc})\end{array}$ & $\begin{array}{l}T_{\mathrm{e}} \\
(\mathrm{K})\end{array}$ & $\begin{array}{l}12+\log (\mathrm{O} / \mathrm{H}) \\
\mathrm{dex}\end{array}$ \\
& $(1)$ & $(2)$ & $(3)$ & $(4)$ & $(5)$ \\
\hline BCLMP 93a & $1: 33: 52.6$ & $30: 39: 08$ & 0.23 & 8700 & $8.53^{a}$ \\
BCLMP 301h & $1: 33: 52.6$ & $30: 45: 03$ & 1.45 & 9200 & 8.60 \\
BCLMP 4a & $1: 33: 59.5$ & $30: 35: 47$ & 1.48 & 9200 & 8.33 \\
M 33-SNR 64 & $1: 34: 00.1$ & $30: 42: 19$ & 0.87 & 8950 & 8.40 \\
\hline
\end{tabular}

Notes. ${ }^{(a)}$ Vílchez et al. 1988 (CC93) adopted $T_{\mathrm{e}}=6000 \mathrm{k}$ obtaining $12+\log (\mathrm{O} / \mathrm{H})=9.02$.

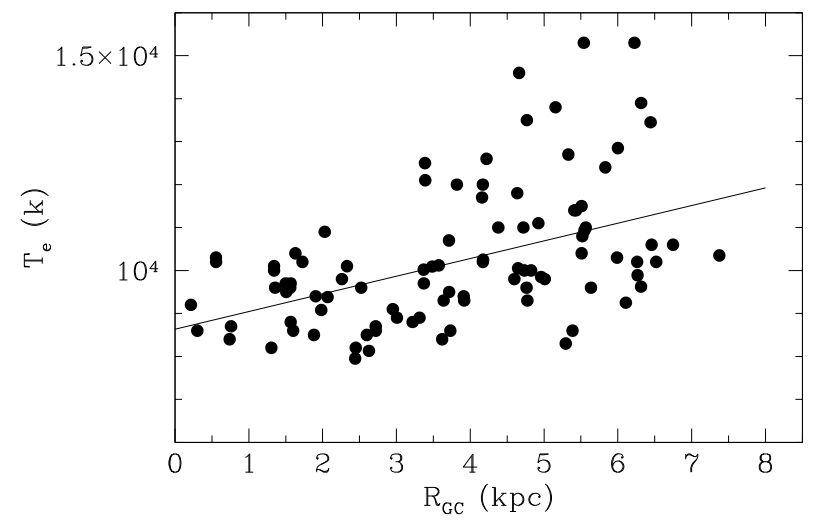

Fig. 7. The radial gradient of $T_{\mathrm{e}}$ for the $\mathrm{H}$ II regions of the present sample and literature data. The continuous line is a mean least square fit.

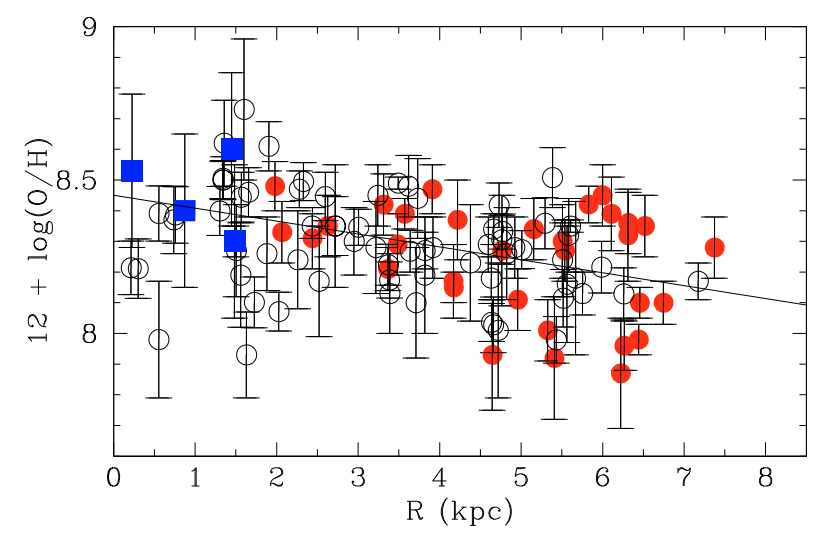

Fig. 8. The $\mathrm{O} / \mathrm{H}$ radial gradient: the symbols for the $\mathrm{H}$ II regions with a positive detection of $T_{\mathrm{e}}$ diagnostic lines are as in Fig. 1, while the filled squares are the regions of Table 7 for which the $T_{\mathrm{e}}$ is extrapolated from Eq. (6). The continuous line is the fit of Eq. (4).

location in the radial gradient is shown in Fig. 8. The sources clearly lie above the average metallicity determined in the centre of our database. It is therefore likely that the adopted criterion for source selection, based on the positive detection of lines for determining the electron temperature, might be responsible for the low metallicity derived in the centre.

In the $1.5 \mathrm{kpc}$ central region, there are $8 \mathrm{H}$ II regions with measured electron temperature having oxygen abundances from $\sim 8$ to $\sim 8.5$ (see Fig. 1), namely B0043b $(\mathrm{O} / \mathrm{H} 8.214)$, B0029 (8.211), B0038b (8.391), B0016 (7.98), B0079c (8.386), B0027b (8.37), B0033b (8.399), B0090 (8.506). Their metal abundance and dispersion are consistent with the hypothesis that we are missing several high-metallicity regions within $1.5 \mathrm{kpc}$ of the centre since they only seem to trace the low metallicity 
side of the distribution present at each given radius. Similarly, this bias partially explains the different gradient of the giant $\mathrm{H}$ II regions: we only include metal-rich $\mathrm{H}$ II regions (with metallicity above a critical value) in the sample if they are very luminous because only these show detectable temperature diagnostic lines.

\section{The chemical evolution of M 33}

The metallicity gradient derived in Sect. 3.1 from our sample of $\mathrm{H}$ II regions characterizes the ISM composition in M 33 at the present time. Together with the metallicity gradient from the PN population (M09), these data allow setting new constraints on current models of galactic chemical evolution.

The model of M07b, specifically designed for M 33, is able to predict the radial distribution of molecular gas, atomic gas, stars, SFR, and the time evolution of the metallicity gradient. In the following, we discuss the modifications needed to reproduce the metallicity gradient of $\mathrm{H}$ II regions and PNe derived in this work and in M09.

\subsection{A revised model of chemical evolution}

The multiphase model adopted by M07b follows the formation and destruction of diffuse gas, clouds, and stars, by means of the simple parameterizations of physical processes (e.g. Ferrini et al. 1992). In particular, the SFR is the result of two processes: cloud-cloud interactions (the dominant process) and star formation induced by the interaction of massive stars on molecular clouds. At variance with other models, the relationship between the SFR surface density, $\Sigma_{\mathrm{SFR}}$, and the molecular gas surface density, $\Sigma_{\mathrm{H}_{2}}$ (the so-called Schmidt law) is thus a by-product of the model and is not assumed. In general, the relation between the surface density of total gas and SFR has a slope of $1.4 \pm 0.1$, but the slope can vary from galaxy to galaxy (Kennicutt 1998a). In the particular case of M 33, a tight correlation exists between the SFR, measured from the FUV emission, and the surface density of molecular gas has a well-defined slope (Verley et al. 2009),

$\Sigma_{\mathrm{SFR}}=A \Sigma_{\mathrm{H}_{2}}^{1.1 \pm 0.1}$.

According to M07b, the best model for M 33 (the so-called $a c$ cretion model, with almost constant infall) suggests a long lasting phase of disk formation resulting from a continuous accretion of intergalactic medium during the galaxy lifetime. We refer to M07b for the general description of the model and of the adopted parameters for M 33. Here we concisely describe the model and only the updated processes and equations in detail.

The galaxy is divided into $N$ coaxial cylindrical annuli with inner and outer galactocentric radii $R_{i}(i=1, N)$ and $R_{i+1}$, respectively, mean radius $R_{i+1 / 2} \equiv\left(R_{i}+R_{i+1}\right) / 2$ and height $h\left(R_{i+1 / 2}\right)$. Each annulus is divided into two zones, the halo and the disk, each made of diffuse gas $g$, clouds $c$, stars $s$, and stellar remnants $r$. The halo component includes the primordial baryonic halo but also the material accreted from the intergalactic medium.

At time $t=0$, all the baryonic mass of the galaxy is in the form of diffuse gas in the halo. At later times, the mass fraction in the various components is modified by several conversion processes: diffuse gas of the halo falls into the disk, diffuse gas is converted into clouds, clouds collapse to form stars and are disrupted by massive stars, and stars evolve into remnants and return a fraction of their mass to the diffuse gas. In this framework, each annulus evolves independently (i.e. without radial mass flows) keeping its total mass (halo + disk) fixed from $t=0$ to $t_{\mathrm{gal}}=13.6 \mathrm{Gyr}$.

The disk of mass $M_{\mathrm{D}}(t)$ is formed by continuous infall from the halo of mass $M_{\mathrm{H}}(t)$ at a rate

$\frac{\mathrm{d} M_{\mathrm{D}}}{\mathrm{d} t}=f M_{\mathrm{H}}$

where $f$ is a coefficient proportional to the inverse of the infall timescale. Clouds condense out of diffuse gas at a rate $\mu$ and are disrupted by cloud-cloud collisions at a rate $H^{\prime}$,

$\frac{\mathrm{d} M_{\mathrm{c}}}{\mathrm{d} t}=\mu M_{\mathrm{g}}^{3 / 2}-H^{\prime} M_{\mathrm{c}}^{2}$,

where $M_{\mathrm{g}}(t)$ is the mass fraction (with respect to the total baryonic mass of the galaxy) of diffuse gas, and $M_{\mathrm{c}}(t)$ was defined above.

Stars form by cloud-cloud interactions at a rate $H$ and by the interactions of massive stars with clouds at a rate $a$,

$\frac{\mathrm{d} M_{\mathrm{s}}}{\mathrm{d} t}=H M_{\mathrm{c}}^{2}+a M_{\mathrm{s}} M_{\mathrm{c}}-D M_{\mathrm{s}}$

where $M_{\mathrm{s}}(t)$ is the mass fraction in stars, $D$ the stellar death rate and $M_{\mathrm{c}}(t)$ as above. All rate coefficients of the model are assumed to be independent of time but are functions of the galactocentric radius $R_{\mathrm{GC}}$ (cf. M07b for their values and radial behaviors).

\subsection{The choice of the stellar yields and the IMF}

The model results are sensitive to the assumed stellar yields. For low- and intermediate-mass stars $\left(M<8 M_{\odot}\right)$, we used the yields by Gavilán et al. (2005). The yields of Marigo (2001) give comparable results without any appreciable difference in the computed gradients of chemical elements produced by intermediate mass stars, such as $N$ with respect to the gradients computed with the yields by Gavilán et al. (2005). For stars in the mass range $8 M_{\odot}<M<35 M_{\odot}$ we adopt the yields by Chieffi \& Limongi (2004). The yields of more massive stars are affected by the considerable uncertainties associated with different assumptions about the modelling of processes like convection, semi-convection, overshooting, and mass loss. Other difficulties arise from the simulation of the supernova explosion and the possible fallback after the explosion, that strongly influences the production of iron-peak elements. It is not surprising then that the results of different authors (e.g. Arnett 1995; Woosley \& Weaver 1995; Thielemann et al. 1996; Aubert et al. 1996) disagree in some cases by orders of magnitude. In our models, we estimate the yields of stars in the mass range $35 M_{\odot}<M<$ $100 M_{\odot}$ by linear extrapolation of the yields in the mass range $8 M_{\odot}<M<35 M_{\odot}$.

Another important ingredient in the chemical evolution model is the initial mass function (IMF). Several works support the idea that the IMF is universal in space and constant in time (Wyse 1997; Scalo 1998; Kroupa 2002), apart from local fluctuations. There are several parameterizations of the IMF (see e.g. Romano et al. 2005, for a complete review), starting from, e.g., Salpeter (1955), Tinsley (1980), Scalo (1986), Kroupa et al. (1993), Ferrini et al. (1990), Scalo (1998), Chabrier (2003). In the following, we test the possibility that the observed flat gradients can be explained in terms of a non-standard IMF. In fact, the magnitude and the slope of chemical abundance gradients are related to the number of stars in each mass range, and so to the IMF. The goal is to reproduce, if possible, the flat gradient supported by recent observations by only varying the IMF. 

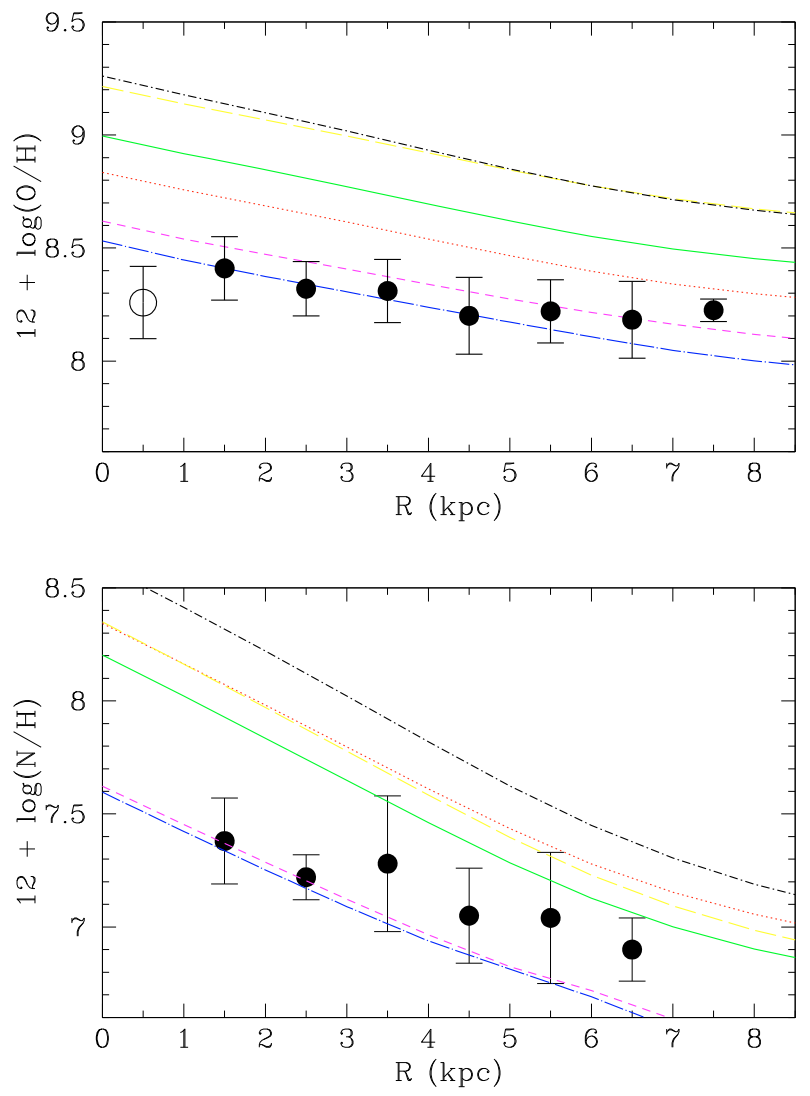

Fig. 9. The oxygen (top panel) and nitrogen (bottom panel) radial gradients of M07b with several parameterization of the IMF: Salpeter (1955) (continuous green line), Tinsley (1980) (dotted red line), Scalo (1986) (dashed magenta line), Scalo 1998) (long dashed yellow line), Ferrini et al. (1990) (long dash-dotted blue line), Chabrier (2003) (dash-dotted black line).

In Fig. 9 we compare the oxygen and nitrogen gradients of $\mathrm{H}$ II regions with present-day abundance profiles from the M07b model. The choice of the IMF does not affect the slope of $\mathrm{O} / \mathrm{H}$ and $\mathrm{N} / \mathrm{H}$ gradients, but simply shifts the abundance profiles to higher (or lower) values according to the amount of stars that produce oxygen (massive stars) or nitrogen (low- and intermediate-mass stars). With the adopted stellar yields, the best fits of the metallicity distribution of M 33 are obtained with the IMF by Ferrini et al. (1990) and Scalo (1986). For the revised model we thus adopt the parameterization of Ferrini et al. (1990). The different slopes of the $\mathrm{O} / \mathrm{H}$ and $\mathrm{N} / \mathrm{H}$ gradients are reproduced fairly well by the chemical evolution model as a natural consequence of the different mass ranges of stellar production, hence timescales, of these two chemical elements (see Sect. 3.2 for a comparison with the measured gradients).

\subsection{Comparison with the observations}

The observational constraints to the model are those described in $\mathrm{M} 07 \mathrm{~b}$, complemented with the $\mathrm{O} / \mathrm{H}$ and $\mathrm{N} / \mathrm{H}$ radial gradients of $\mathrm{H}$ II regions and PNe from this work and M09, respectively, and the radial profile of the SFR determined by Verley et al. (2009) from far ultraviolet (FUV) observations corrected for extinction. For $\mathrm{H}$ II regions we used the gradient derived from the whole population (see Eq. (4)), without any distinction in terms of size and brightness, but excluding the central $1 \mathrm{kpc}$. Giant

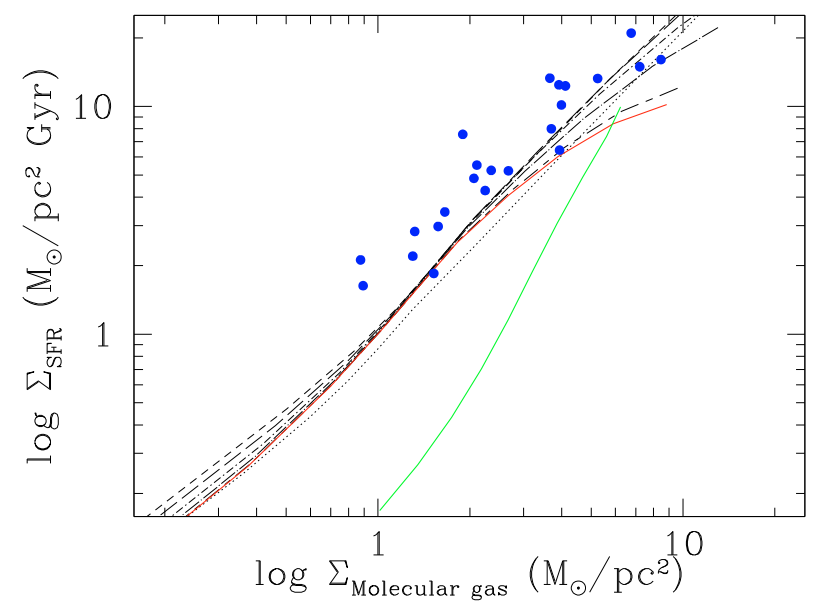

Fig. 10. The SFR derived from the UV emission corrected for extinction by Verley et al. (2009) vs. the surface density Schmidt law: filled circles (blue) are the molecular gas averaged in bins $1 \mathrm{kpc}$ each (Corbelli 2003; curves represent the model at $0.5 \mathrm{Gyr}$ from the disk formation (dotted curve), 2 (dashed curve), 3 (long-dashed curve), 5 (dot-dashed curve), 8 (long dash-dotted curve), 12 (long-short dashed curve), and at 13.6 Gyr (solid red curve); the solid green curve is the model by M07b at $13.6 \mathrm{Gyr}$.

$\mathrm{H}$ II regions might not be representative of the current ISM abundance owing their possible chemical self-enrichment.

In Figs. 10 and 11 we show the radial surface density of molecular gas (from the single dish observations of Corbelli 2003, averaged over $1 \mathrm{kpc}$ bins) and the SFR as a function of the surface density of molecular gas (Verley et al. 2009), respectively. Both figures show the predictions of the model of M07b at $t=t_{\mathrm{gal}}$. Clearly, the model of M07b in its original formulation is unable to reproduce these constraints. We have therefore considered other parameterizations of the star formation process by varying the radial dependence of the SFE, represented by the coefficient $H$.

Our experiments show that, to reproduce the observed behaviour of the radial gas distribution and the observed Schmidt law, is necessary to increase the efficiency of star formation at large radii. This can be accomplished in many ways. In the previous version of the model (M07b) $H$ decreased with $R_{\mathrm{GC}}$ to consider a geometrical correction resulting from how large galactocentric distances correspond to larger volumes (see Ferrini et al. 1994). In the present work we assume that $H$ is constant with radius, thus implying that the star formation efficiency increases linearly with galactocentric radius. In Sect. 5.4 we describe the observational evidence in support this assumption in M 33.

Assuming that $H$ is spatially constant, we obtain the results shown in Figs. 10 and 11 at $t=0.5,2,3,5,8,12,13.6$ Gyr. The relationship between molecular gas and SFR predicted by the modified M07b model is more or less constant with time and in good agreement with the data. The predicted Schmidt law (at the present time) has an average exponent $\sim 1.2$, in agreement with the observations, whereas the original M07b model produces a Schmidt law with a higher exponent, $\sim 2.2$. The better agreement with the observations obtained with the revised M07b model suggests that M 33 is more efficient in forming stars than normal local Universe spiral galaxies, in particular in its outer regions. 


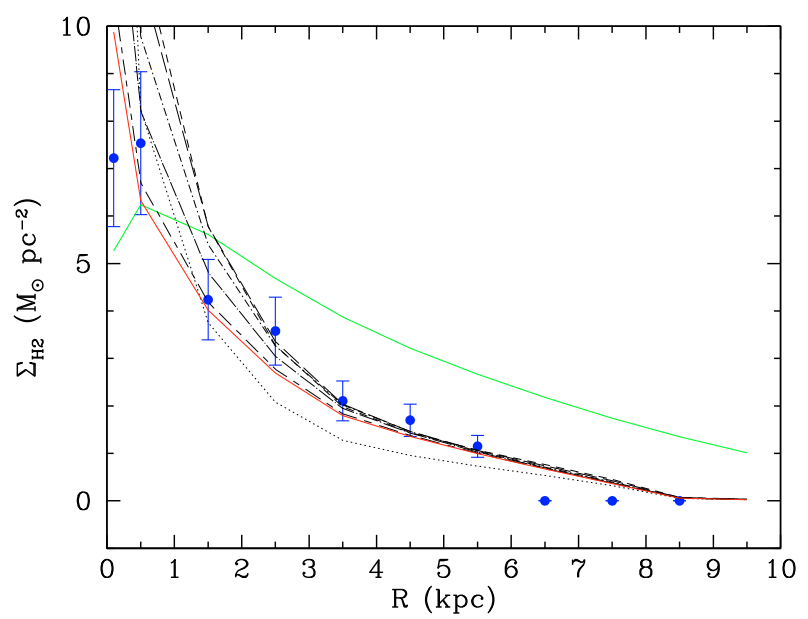

Fig. 11. The radial surface density of molecular gas: filled circles (blue) are the observed molecular gas averaged in bins $1 \mathrm{kpc}$ wide. Model curves have the same symbols as in Fig. 10.

\subsection{Flat gradients and SF efficiency}

Is there evidence of flat gradients in other galaxies? How can they be explained? A flat gradient in the outer regions of our Galaxy has been observed by several authors (e.g., Yong et al. 2005; Carraro et al. 2007; Sestito et al. 2007) using different metallicity tracers (e.g., Cepheids, open clusters). However, other tracers, such as $\mathrm{PNe}$, do not show this behaviour, indicating flat gradients across the whole disk (cf. Stanghellini et al. 2006; Perinotto \& Morbidelli 2006). The outer Galactic plateau might be a phenomenon similar to the flat gradient of M 33. Since M 33 is less massive than the MW, its halo collapse phase, responsible for the steep gradient in the inner regions $\left(R_{\mathrm{GC}}<11-12 \mathrm{kpc}\right)$ of our Galaxy (cf. Magrini et al. 2009b), is less marked. Thus in M 33 the difference between the inner and outer gradients is less evident than in the MW. The flat metallicity distribution of the MW at large radii has been explained with several chemical evolution models, among them those by Andrievsky et al. (2004), Chiappini et al. (2001) model C, and Magrini et al. (2009b). Andrievsky et al. (2004) explain the flat metallicity distribution beyond $11-12 \mathrm{kpc}$ when assuming that the SFR is a combination of two components: one proportional to the gas surface density, and the other depending on the relative velocity of the interstellar gas and spiral arms. With these assumptions they explain why the breaking point in the slope of the gradient and the consequent outer flattening, occur around the co-rotational radius. Chiappini et al. (2001) assume two main accretion episodes in the lifetime of the Galaxy, the first one forming the halo and bulge and the second one forming the thin disk. Their model $\mathrm{C}$ is the one able to reproduce a flat gradient in the external regions, assuming that there is no threshold in the gas density during the halo/thickdisk phase, and thus allowing the formation of the outer plateau from infalling gas enriched in the halo. Finally, Magrini et al. (2009b) reproduce the Galactic gradient thanks to the radial dependence of the infall rate (exponentially decreasing with radius) and with the radial dependence of the star and cloud formation processes. To reproduce a completely flat gradient in the outer regions would, however, require additional accretion of gas uniformly falling onto the disk, which would result in inconsistent behaviour of the current SFR.

Why do we use a radially increasing SFE to reproduce the flat metallicity gradient of M 33? M 33 in general is quite different from large spiral galaxies in terms of SF. A comparison of the SFR to the $\mathrm{H}_{2}$ mass shows that M 33, like the intermediate redshift galaxies, has a significantly higher SFE than large local Universe spirals. There is also observational evidence that the SFE varies with radius in M 33. Kennicutt (1998b), Wong \& Blitz (2002), and Murgia et al. (2002) find a molecular gas depletion timescale (inversely proportional to the SFE) that varies radially, decreasing by a factor $\sim 2-3$. Also Gardan et al. (2007) from the CO to SFR ratio also find a dependence of the depletion timescale on radius, decreasing of a factor $\sim 2$ over $4 \mathrm{kpc}$. These observations prompted us to model the chemical evolution of M 33 taking SF processes depending on radius into account. The general assumption of our model is that the SF is driven by cloud collisions. Increasing the efficiency of this process with radius does not imply that cloud-cloud collisions are more efficient in the outer regions, but that additional processes may contribute to the SF in the peripheral regions.

\subsection{Implications for the metallicity gradient and its evolution}

The introduction of more realistic SF process in M 33 has significant consequences on its metallicity gradient and evolution. As described by M09, oxygen modification (destruction or creation) does not occur in the PN population of M 33. Thus oxygen, the best-determined element in nebular optical spectroscopy, can be safely used as a tracer of the ISM composition at the epoch of the formation of PN progenitors. Most PNe in M 33 are older than $0.3 \mathrm{Gyr}$, and probably much older, up to an age of $10 \mathrm{Gyr}$.

The metallicity gradient of PNe, including only those with progenitor stars older than $0.3 \mathrm{Gyr}$ and excluding PNe located in the first kpc from the centre, is

$$
12+\log (\mathrm{O} / \mathrm{H})=-0.040( \pm 0.014) R_{\mathrm{GC}}+8.43( \pm 0.06)
$$

As discussed above, the whole sample of H II regions is representative of the current ISM composition. The $\mathrm{H}$ II region and PN gradients in the same radial region, $1 \mathrm{kpc} \lessgtr R_{\mathrm{GC}} \lesssim 8 \mathrm{kpc}$, are indistinguishable within the uncertainties in their slopes, with a slightly translation, $\sim 0.1 \mathrm{dex}$, towards higher metallicity in the $\mathrm{H}$ II region sample. This means that very little evolution of metallicity has occurred in the past few Gyr.

In Fig. 12 we show the observed metallicity gradient of M 33 together with the results of the present model (top panel) and of the original model of M07b (bottom panel). For a better comparison, the oxygen abundances of the samples of $\mathrm{H}$ II regions and disk PNe have been averaged over bins of $1 \mathrm{kpc}$ each. This allows us to highlight the translation towards the lower metallicity of the PNe. The curves correspond to the present and to 5 Gyr ago (8.6 Gyr from the disk formation, assuming an age of 13.6 Gyr for M 33), i.e., approximately the average period of formation of the PN progenitors. The present model predicts a higher SF in the outer regions, reproducing the observed flatness of the oxygen gradient at large radii better than the M07b model. In addition, the evolution of the metallicity gradient predicted by the new model is consistent with the observations: a negligible change in the slope and a small translation to higher metallicities. Finally, we see that the assumed dependence of the SFE with galactocentric radius, required to reproduce the metallicity evolution of M 33, takes place always as in M07b with a slow building up of the disk of M 33 by accretion from an intergalactic medium or halo gas.

For completeness we also show the $\mathrm{N} / \mathrm{H}$ radial gradient of the sample of $\mathrm{H}$ II regions. For this element the temporal evolution cannot be inferred using PN abundances, since PNe modify 


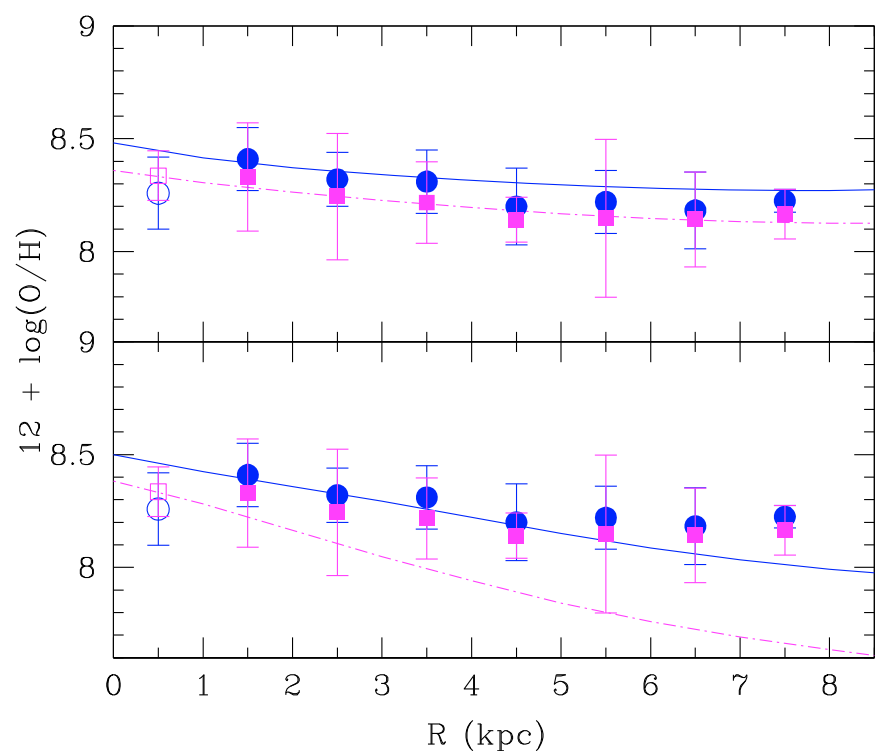

Fig. 12. The O/H radial gradient evolution. Top panel: filled circles (blue) are the $\mathrm{H}$ II region oxygen abundances from the dataset described in Sect. 2, averaged over radial bins, each $1 \mathrm{kpc}$ wide; filled squares (magenta) are averaged non-type I PN oxygen abundances by M09. The gradient 5 Gyr ago (dashed curve) and now (continuous curve) as predicted by the present model. Bottom panel: the same as in the top panel but for the accretion model by M07b.

their nitrogen composition during their lifetime. The nitrogen abundances have been averaged over bins of $1 \mathrm{kpc}$ each. The observed $\mathrm{N} / \mathrm{H}$ gradient is significantly steeper than the oxygen gradient, as predicted by the model.

The time evolution of the metallicity gradient is strictly correlated to the inside-out growth of the disk. In a recent work, Williams et al. (2009) presented the resolved photometry of four fields located at different galactocentric distances, from 0.9 to $6.1 \mathrm{kpc}$. Their photometry provides a detailed census of stellar populations and their ages at different radii. They find that the percentage of the stellar mass formed prior to $z=1$ ( $\sim 8 \mathrm{Gyr}$ ago $)$ changes from $\sim 71 \%$ in the innermost field at $0.9 \mathrm{kpc}$ from the centre to $\sim 16 \%$ in the outermost SF region at $6.1 \mathrm{kpc}$. We thus compare these results with the cumulative SF predicted by our model at different ages. The agreement is good in the central field, where model and observations both have about $70 \%$ of the stellar mass already formed $8 \mathrm{Gyr}$ ago. Also at $2.5 \mathrm{kpc}$, corresponding to their second field, the observed stellar mass formed prior to $z=1$ is about $50 \%$ and the model results give $47 \%$. In the outermost fields, at 4.3 and $6.1 \mathrm{kpc}$, the agreement is not as good, since the observations show that $20 \%$ and $16 \%$ of stars formed before $8 \mathrm{Gyr}$ ago, while the model predicts $\sim 44 \%$ for both fields. With the model by M07b, the situation is almost unvaried in the inner regions, while in the outer regions there is a slightly lower percentage of stars formed before $8 \mathrm{Gyr}$ ago, $\sim 33 \%$. The recent evolution of the outermost SF regions of M 33 can be explained by an accretion of material in the peripheral regions at recent epochs. In our model, the scalelength of the disk is constant, and the accretion in the outer regions is a continuous process. Thus, we are able to reproduce the integrated SF at any radius and its by-product, the metallicity, but not to reproduce its temporal behaviour if it is dominated by stochastic events, such as, e.g., sporadic massive accretion of gas and tidal interactions. Finally, outside the SF area of the disk, the age of populations of the outer disk/halo show an age increase with radius (Barker et al. 2007a,b). This agrees well with our model

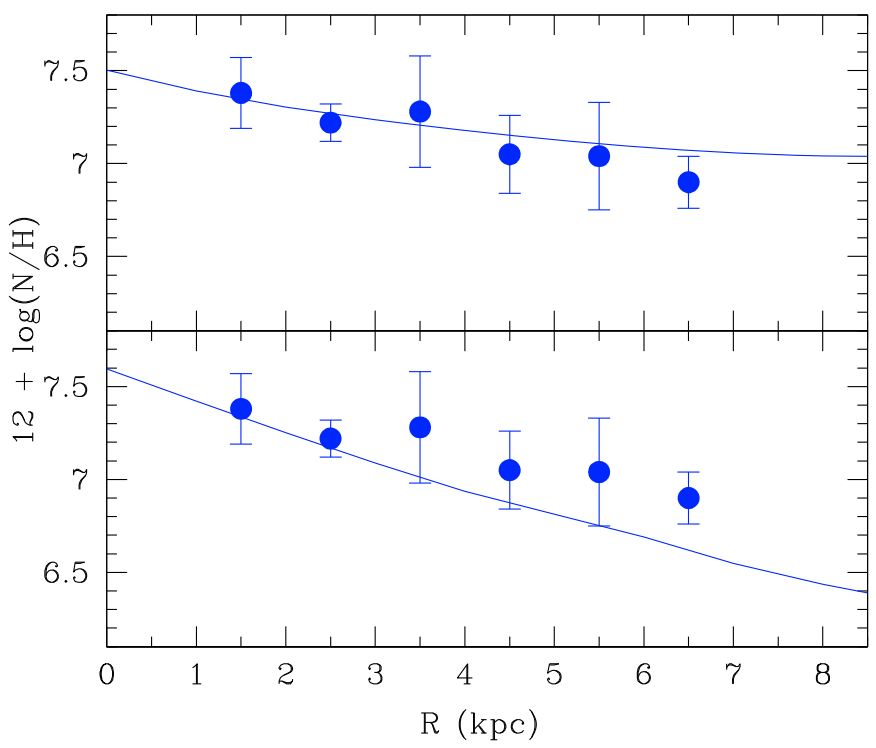

Fig. 13. The N/H radial gradient. Top panel: filled circles (blue) are the $\mathrm{H}$ II region nitrogen abundances from the dataset described in Sect. 2, averaged over radial bins, each $1 \mathrm{kpc}$ wide. The gradient at the present time as predicted by the present model is the continuous curve. Bottom panel: the same as in the top panel but for the accretion model by M07b.

results: there is a slightly larger number of old stars beyond $8 \mathrm{kpc}$ than in the peripheral part of the SF disk.

\section{Summary and conclusions}

We have studied the chemical evolution of M 33 by means of new spectroscopic observations of $\mathrm{H}$ II regions, together with literature data of $\mathrm{H}$ II regions and $\mathrm{PNe}$. We derived the radial oxygen gradient in the range from 1 to $\sim 8 \mathrm{kpc}$. Its slope is $-0.044 \pm$ $0.009 \mathrm{dex} \mathrm{kpc}^{-1}$. We excluded the central $1 \mathrm{kpc}$ region from the gradient because of its low metallicity. In fact, the 2D metallicity map is off-centre, with a peak in the southern arm at 1-2 kpc from the centre. We measured the highest metallicity gradient in bright regions which are not located in the centre of M 33 . We explained this effect with a bias in the measurement of nebular abundances towards low metallicity in the central regions and/or with SF bursts which had not had time to mix azimuthally. At a galactocentric distance of about $1.5 \mathrm{kpc}$, the spread of metal abundances is much greater than the dispersion along the average gradient, and it is possibly related to the presence of a bar.

We analysed and discussed the metallicity gradient of giant $\mathrm{H}$ II regions, i.e. the largest regions with high surface brightness. We find it steeper than the average of the sample gradient, in agreement with the early spectroscopic studies of $\mathrm{H}$ II regions in M 33, based mainly on the brightest objects.

We compared the metallicity gradients of $\mathrm{H}$ II regions and of PNe, obtained with the same set of observations and analysis techniques. We find a substantially unchanged slope of the gradient, and an overall increase in metallicity with time. We can explain the slow evolution of the metallicity gradient from the present to the birth of the PN progenitors with a chemical evolution accretion model, as in M07b, if the SFE is higher at larger radii.

\section{Appendix A: Comparison with the literature}

To assess the quality of the MMT observations we compared the oxygen abundance in the control sample with those available in 
A\&A 512, A63 (2010)

Table A.1. Comparison of oxygen measurements of our control sample with literature $\mathrm{O} / \mathrm{H}$.

\begin{tabular}{|c|c|c|c|c|c|c|}
\hline \multirow{2}{*}{$\begin{array}{l}\text { ID } \\
\text { (1) }\end{array}$} & \multicolumn{6}{|c|}{$12+\log (\mathrm{O} / \mathrm{H})$} \\
\hline & $\begin{array}{l}\text { M07a } \\
\text { (2) }\end{array}$ & $\begin{array}{l}\text { RS08 } \\
\text { (3) }\end{array}$ & $\begin{array}{l}\text { C06 } \\
\text { (4) }\end{array}$ & $\begin{array}{l}\text { KA81 } \\
(5)\end{array}$ & $\begin{array}{l}\text { V88 } \\
(6)\end{array}$ & $\begin{array}{l}\text { pw } \\
(7)\end{array}$ \\
\hline LGCHII2 & $8.25 \pm 0.06$ & & & & & $8.10 \pm 0.05$ \\
\hline LGCHII3 & $8.24 \pm 0.05$ & & & & & $8.42 \pm 0.06$ \\
\hline BCLMP289 & $8.25 \pm 0.13$ & & & & & $8.35 \pm 0.12$ \\
\hline BCLMP218 & $8.25 \pm 0.05$ & $8.16 \pm 0.06$ & & & & $8.17 \pm 0.12$ \\
\hline MA1 & & & $8.24 \pm 0.06$ & & & $8.28 \pm 0.15$ \\
\hline BCLMP290 & & & $8.21 \pm 0.06$ & & & $8.37 \pm 0.13$ \\
\hline IC 132 & $8.08 \pm 0.04$ & & & & & $7.98 \pm 0.05$ \\
\hline BCLMP45 & $8.49 \pm 0.04$ & & & & & $8.48 \pm 0.08$ \\
\hline BCLMP670 & $8.28 \pm 0.07$ & & & & & $8.29 \pm 0.07$ \\
\hline MA2 & & $8.33 \pm 0.08$ & & 8.38 & $8.44 \pm 0.15$ & $8.31 \pm 0.10$ \\
\hline BCLMP691 & & & $8.26 \pm 0.02$ & & & $8.42 \pm 0.06$ \\
\hline IC 131 & & & & & $8.43 \pm 0.03$ & $8.47 \pm 0.08$ \\
\hline IC 133 & & $8.23 \pm 0.05$ & & & & $8.27 \pm 0.08$ \\
\hline BCLMP745 & & & $8.07 \pm 0.10$ & & & $7.93 \pm 0.10$ \\
\hline
\end{tabular}

References. M07a - Magrini et al. (2007a); RS08 - Rosolowsky \& Simon (2008); C06 - Crockett et al. (2006); KW81 - Kwitter \& Aller (1981); V88 - Vilchez et al. (1988); present work (pw).

the literature. Oxygen is in fact the most widely used element in emission-line optical spectroscopy because it gives accurate results for determining the metallicity. This is because i) many ionization stages are present in the optical range ([O I], [O II], [O III]), and the corrections for unseen ionization stages are often not needed; ii) a direct measurement of its electron temperatures, $T_{\mathrm{e}}[\mathrm{O} \mathrm{III}]$ and $T_{\mathrm{e}}[\mathrm{O} \mathrm{II}]$, is possible. For each source in the control sample, we show in Table A.1 the oxygen abundance determined by different authors (Rosolowsky \& Simon 2008; Magrini et al. 2007a; Crockett et al. 2006; Vilchez et al. 1988; Kwitter \& Aller 1981) and in this paper. In Cols. 2 to 6 we report the literature $\mathrm{O} / \mathrm{H}$ and our measurements in $\mathrm{Col}$. 7. For $\mathrm{H}$ II regions with multiple determinations we computed the weighted average of all measurements. The agreement is reasonably good for all sources, since our values are consistent, within the errors, with those available in the literature. The average $\mathrm{O} / \mathrm{H}$ abundance of the $14 \mathrm{H}$ II regions agrees. The average dispersion between our values and previous determinations is only 0.09 dex.

Acknowledgements. We warmly thank J. Vilchez for interesting discussion on the argument. We thank R. Walterbos for allowing us to use his $\mathrm{H} \alpha$ calibrated map of M 33. We thank D. Fabricant for making Hectospec available to the community, and the Hectospec instrument team and MMT staff for their expert help in preparing and carrying out the Hectospec observing runs. We thank N. Caldwell, D. Ming, and their team for the help during the data reduction. L.S. acknowledges the hospitality of the Observatoire de Paris where a part of this work was developed.

\section{References}

Afflerbach, A., Churchwell, E., \& Werner, M. W. 1997, ApJ, 478, 190

Andrievsky, S. M., Luck, R. E., Martin, P., \& Lèpine, J. R. D. 2004, A\&A, 413, 159

Arnett, D. 1995 ARA\&A, 33, 115

Asplund, M., Grevesse, N., \& Sauval, A. J. 2005, ASPC, 336, 25

Aubert, O., Prantzos, N., \& Baraffe, I. 1996, A\&A, 312, 845

Barker, M. K., Sarajedini, A., Geisler, D., Harding, P., \& Schommer, R. 2007a, AJ, 133, 1125

Barker, M. K., Sarajedini, A., Geisler, D., Harding, P., \& Schommer, R. 2007b, AJ, 133, 1138

Benjamin, R. A., Skillman, E. D., \& Smits, D. P. 1999, ApJ, 514, 307

Bonanos, A. Z., Stanek, K. Z., Kudritzki, R. P., et al. 2006, ApJ, 652, 313

Boulesteix, J., Courtes, G., Laval, A., Monnet, G., \& Petit, H. 1974, A\&A, 37, 33

Bresolin, F. 2007, ApJ, 656, 186

Bresolin, F., Gieren, W., Kudritzki, R.-P., et al. 2009, ApJ, 700, 309
Brunthaler, A., Reid, M. J., Falcke, H., Greenhill, L. J., \& Henkel, C. 2005, Science, 307, 1440

Chabrier, G. 2003, PASP, 115, 763

Chiappini, C., Matteucci, F., \& Romano, D. 2001, ApJ, 554, 1044

Chieffi, A., \& Limongi, M. 2004, ApJ, 608, 405

Carraro, G., Geisler, D., Villanova, S., Frinchaboy, P. M., \& Majewski, S. R. 2007, A\&A, 476, 217

Clegg, R. E. S. 1987, MNRAS, 229, 31

Colin, J., \& Athanassoula, E. 1981, A\&A, 97, 63

Corbelli, E. 2003, MNRAS, 342, 199

Corbelli, E., \& Walterbos, R. A. M. 2007, ApJ, 669, 315

Corradi, R. L. M., \& Magrini, L. 2006, in Planetary Nebulae Beyond the Milky Way, ESO Astrophys. Symp., 36

Courtes, G., Petit, H., Petit, M., Sivan, J.-P., \& Dodonov, S. 1987, A\&A, 174, 28 Crockett, N. R., Garnett, D. R., Massey, P., \& Jacoby, G. 2006, ApJ, 637, 741

Devereux, N., Duric, N., \& Scowen, P. A. 1997, AJ, 113, 236

Dopita, M. A. 1991, IAUS, 148, 393

Engargiola, G., Plambeck, R. L., Rosolowsky, E., \& Blitz, L. 2003, ApJS, 149, 343

Fabricant, D., Fata, R., Roll, J., et al. 2005, PASP, 117, 1411

Ferrini, F., Penco, U., \& Palla, F. 1990, A\&A, 231, 391

Ferrini, F., Matteucci, F., Pardi, C., \& Penco, U. 1992, ApJ, 387, 138

Ferrini, F., Mollá, M., Pardi, M. C., \& Diaz, A. I. 1994, ApJ, 427, 745

Freedman, W. L., Wilson, C. D., \& Madore, B. F. 1991, ApJ, 372, 455

Gardan, E., Braine, J., Schuster, K. F., Brouillet, N., \& Sievers, A. 2007, A\&A, 473,91

Garnett, D. R., Shields, G. A., Skillman, E. D., Sagan, S. P., \& Dufour, R. J. 1997, ApJ, 489, 63

Gavilán, M., Buell, J. F., \& Mollá, M. 2005, A\&A, 432, 861

Gordon, S. M., Duric, N., Kirchner, R. P., Goss, W. M., \& Viallefond, F. 1999, ApJS, 120, 247

Herrero, A., Lennon, D. J., Vílchez, J. M., Kudritzki, R. P., \& Humphreys, R. H. 1994, A\&A, 287, 885

Holmberg, E. 1958, Lund Medd. Astron. Obs. Ser. II, 136, 1

Kennicutt, R. C. 1998, ApJ, 498, 541

Kennicutt, R. C., Jr. 1998, ARA\&A, 36, 189

Kingsburgh, R. L., \& Barlow, M. J. 1994, MNRAS, 271, 257

Kroupa, P. 2002, Science, 295, 82

Kroupa, P., Tout, C. A., \& Gilmore, G. 1993, MNRAS, 262, 545

Kwitter, K. B., \& Aller, L. H. 1981, MNRAS, 195, 939

Magrini, L., Vilchez, J. M., Mampaso, A., Corradi, R. L. M., \& Leisy, P. 2007a, A\&A, 470, 865 (M07a)

Magrini, L., Corbelli, E., \& Galli, D. 2007b, A\&A, 470, 843 (M07b)

Magrini, L., Stanghellini, L., \& Villaver, E. 2009a, ApJ, 696, 729 (M09)

Magrini, L., Sestito, P., Randich, S., \& Galli, D. 2009b, A\&A, 494, 95

Marigo, P. 2001, A\&A, 370, 194

Maraston, C. 2005, MNRAS, 362, 799

Massey, P., \& Johnson, O. 1998, ApJ, 505, 793

Massey, P., Strobel, K., Barnes, J. V., \& Anderson, E. 1988, ApJ, 328, 315

Mathis, J. S. 1990, ARA\&A, 28, 37 
McCarthy, J. K., Lennon, D. J., Venn, K. A., \& Kudritzki, R.-P. 1995, ApJ, 455, 135

Minniti, D., Olszewski, E. W., \& Rieke, M. 1993, ApJ, 410, L79

Monteverde, M. I., Herrero, A., Lennon, D. J., \& Kudritzki, R.-P. 1997, ApJ, 474,107

Monteverde, M. I., Herrero, A., \& Lennon, D. J. 2000, ApJ, 545, 813

Murgia, M., Crapsi, A., Moscadelli, L., \& Gregorini, L. 2002, A\&A, 385, 412

Nagao, T., Maiolino, R., \& Marconi, A. 2006, A\&A, 459, 85

Osterbrock, D. E., \& Ferland, G. J. 2006, Astrophysics of Gaseous Nebulae and Active Galactic Nuclei, 2nd. edn., ed. D. E. Osterbrock, \& G. J. Ferland (Sausalito, CA: University Science Books), Sky and Telescope, 78

Perinotto, M., \& Morbidelli, L. 2006, MNRAS, 372, 45

Press, W. H., Teukolsky, S. A., Vetterling, W. T., \& Flannery, B. F. 1992, Numerical Recipes in FORTRAN: The art of scientific computing, 2nd edn. Regan, M. W., \& Vogel, S. N. 1994, ApJ, 434, 536

Relaño, M., \& Kennicutt, R. C. 2009, ApJ, 699, 1125

Romano, D., Chiappini, C., Matteucci, F., \& Tosi, M. 2005, A\&A, 430, 491

Rosolowsky, E., \& Simon, J. D. 2008, ApJ, 675, 1213

Rubin, R. H., Simpson, J. P., Colgan, S. W. J., et al. 2008, MNRAS, 387, 45

Salpeter, E. E. 1955, ApJ, 121, 161

Sarajedini, A., Barker, M. K., Geisler, D., Harding, P. \& Schommer, R. 2006, AJ, 132,1361

Scalo, J. 1986, Fundamentals of Cosmic Physics, 11, 1

Scalo, J. 1998, ASPC, 142, 201

Schmidt, M. 1959, ApJ, 129, 243

Searle, L. 1971, ApJ, 168, 327
Sestito, P., Randich, S., \& Bragaglia, A. 2007, A\&A, 465, 185 Skrutskie, M. F., Cutri, R. M., Stiening, R., et al. 2006, AJ, 131, 1163 Simon, J. D., \& Rosolowsky, E. 2008, ASPC, 396, 23

Shaw, R. A., \& Dufour, R. J. 1994, ASPC, 61, 327

Smith, H. E. 1975, ApJ, 199, 591

Stasińska, G. 2005, A\&A, 434, 507

Stanghellini, L., Guerrero, M. A., Cunha, K., Manchado, A., \& Villaver, E. 2006, ApJ, 651, 898

Stephens, A. W., \& Frogel, J. A. 2002, AJ, 124, 2023

Thielemann, F.-K., Nomoto, K., \& Hashimoto, M.-A. 1996, ApJ, 460, 408

Tinsley, B. M. 1981, Fundamentals of Cosmic Physics, 5, 287

Thilker, D. A., Hoopes, C. G., Bianchi, L., et al. 2005, ApJ, 619, L67

Urbaneja, M. A., Herrero, A., Kudritzki, R.-P., et al. 2005, ApJ, 635, 311

Vivian, U., Urbaneja, M. A., Kudritzki, R.-P., et al. 2009, ApJ, 704, 1120

Venn, K. A., McCarthy, J. K., Lennon, D. J., \& Kudritzki, R. P. 1998, ASPC, 147,54

Verley, S., Corbelli, E., Giovanardi, C., \& Hunt, L. K. 2009, A\&A, 493, 453

Vílchez, J. M., Pagel, B. E. J., Diaz, A. I., Terlevich, E., \& Edmunds, M. G. 1988, MNRAS, 235, 633

Williams, B. F., Dalcanton, J. J., Dolphin, A. E., Holtzman, J., \& Sarajedini, A. 2009, ApJ, 695, L15

Willner, S. P., \& Nelson-Patel, K. 2002, ApJ, 598, 679

Wilson, C. D., \& Scoville, N. 1989, ApJ, 347, 743

Wong, T., \& Blitz, L. 2002, ApJ, 569, 157

Woosley, S. E., \& Weaver, T. A. 1995, ApJS, 101, 181

Wyse, R. F. G. 1997, ApJ, 490, 69

Yong, D., Carney, B. W., \& de Almeida, L. 2005, AJ, 130, 597 